\title{
MULTI-WAVELENGTH OBSERVATIONS OF BLAZAR AO 0235+164 IN THE 2008-2009 FLARING STATE
}

M. AckermanN ${ }^{1}$, M. Ajello ${ }^{2}$, J. Ballet ${ }^{3}$, G. Barbiellini ${ }^{4,5}$, D. Bastieri ${ }^{6,7}$, R. Bellazzini $^{8}$, R. D. Blandford ${ }^{2}$, E. D. Bloom ${ }^{2}$, E. Bonamente ${ }^{9,10}$, A. W. Borgland ${ }^{2}$, E. Bottacini ${ }^{2}$, J. Bregeon ${ }^{8}$, M. Brigida ${ }^{11,12}$, P. Bruel ${ }^{13}$, R. BuehleR ${ }^{2}$, S. Buson ${ }^{6,7}$, G. A. Caliandro ${ }^{14}$, R. A. Cameron ${ }^{2}$, P. A. Caraveo ${ }^{15}$, J. M. Casandian ${ }^{3}$, E. Cavazzuti ${ }^{16}$, C. Cecchi ${ }^{9,10}$,

E. Charles ${ }^{2}$, A. Chekhtman ${ }^{17,89}$, J. Chiang ${ }^{2}$, S. Ciprini ${ }^{18,10}$, R. Claus ${ }^{2}$, J. Cohen-Tanugi ${ }^{19}$, S. Cutini ${ }^{16}$, F. D' Ammando ${ }^{20,21}$, F. de Palma ${ }^{12}$, C. D. Dermer 22 , E. do Couto e Silva ${ }^{2}$, P. S. Drell ${ }^{2}$, A. Drlica-Wagner ${ }^{2}$, R. Dubois ${ }^{2}$, C. Favuzzi ${ }^{11,12}$, S. J. Fegan ${ }^{13}$, E. C. Ferrara ${ }^{23}$, W. B. Focke ${ }^{2}$, P. Fortin ${ }^{13}$, L. FuhrmanN ${ }^{24}$, Y. Fukazawa ${ }^{25}$, P. Fusco ${ }^{11,12}$, F. Gargano ${ }^{12}$, D. Gasparrini ${ }^{16}$, N. Gehrels ${ }^{23}$, S. Germani ${ }^{9}, 10$, N. Giglietto ${ }^{11,12}$, P. Giommi ${ }^{16}$, F. Giordano ${ }^{11,12}$, M. Giroletti ${ }^{26}$,

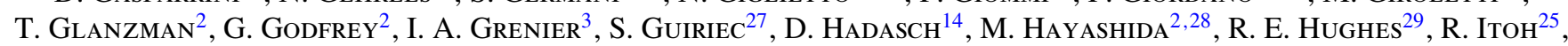

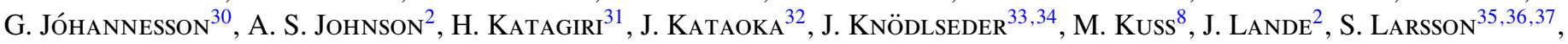
S.-H. LeE ${ }^{38}$, F. Longo ${ }^{4,5}$, F. Loparco ${ }^{11,12}$, B. LotT ${ }^{39}$, M. N. Lovellette ${ }^{22}$, P. Lubrano ${ }^{9,10}$, G. M. Madejski ${ }^{2}$, M. N. Mazziotta ${ }^{12}$, J. E. McEnery ${ }^{23,40}$, J. Mehault ${ }^{19}$, P. F. Michelson ${ }^{2}$, W. Mitthumsiri ${ }^{2}$, T. Mizuno ${ }^{25}$, C. Monte ${ }^{12}$, M. E. Monzani ${ }^{2}$, A. Morsellit ${ }^{41}$, I. V. Moskalenko ${ }^{2}$, S. Murgia ${ }^{2}$, M. NAUmann-Godo ${ }^{3}$, S. Nishino ${ }^{25}$, J. P. Norris ${ }^{42}$, E. Nuss $^{19}$, T. Ohsugi ${ }^{43}$, A. OKumura ${ }^{2,44}$, N. Omodei ${ }^{2}$, E. Orlando ${ }^{2,45}$, M. Ozaki ${ }^{44}$, D. Paneque ${ }^{46,2}$, J. H. Panetta ${ }^{2}$, V. Pelassa ${ }^{27}$, M. Pesce-Rollins ${ }^{8}$, M. Pierbattista ${ }^{3}$, F. Piron ${ }^{19}$, G. Pivato $^{7}$, T. A. Porter ${ }^{2}$, S. Rainò ${ }^{12}$, R. Rando ${ }^{6,7}$,

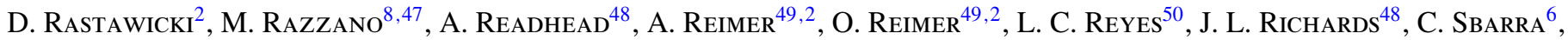
C. Sgrò ${ }^{8}$, E. J. Siskind ${ }^{51}$, G. Spandre ${ }^{8}$, P. Spinelli ${ }^{11,12}$, A. Szostek ${ }^{2}$, H. Takahashi ${ }^{43}$, T. Tanaka ${ }^{2}$, J. G. Thayer ${ }^{2}$, J. B. ThaYeR ${ }^{2}$, D. J. Thompson ${ }^{23}$, M. Tinivella ${ }^{8}$, D. F. Torres ${ }^{14,52}$, G. Tosti ${ }^{9,10}$, E. Troja ${ }^{23,90}$, T. L. Usher ${ }^{2}$, J. Vandenbroucke ${ }^{2}$, V. Vasileiou ${ }^{19}$, G. Vianello ${ }^{2,53}$, V. Vitale ${ }^{41,54}$, A. P. Waite ${ }^{2}$, B. L. Winer ${ }^{29}$, K. S. Wood ${ }^{22}$, Z. YANG ${ }^{35,36}$, S. ZIMMER ${ }^{35,36}$

(THE Fermi-LAT COlLaboRATiOn)

R. MODERSKI ${ }^{55}$, K. NALEWAJKo ${ }^{55,56}$, M. SiKora ${ }^{55}$

AND

M. Villata ${ }^{57}$, C. M. Raiteri ${ }^{57}$, H. D. Aller ${ }^{58}$, M. F. Aller ${ }^{58}$, A. A. Arkharov ${ }^{59}$, ${\text { E. } \text { Benítez }^{60} \text {, A. Berdyugin }}^{61}$, D. A. Blinov ${ }^{59}$, M. Boettcher ${ }^{62}$, O. J. A. Bravo Calle ${ }^{63}$, C. S. Buemi ${ }^{64}$, D. Carosati ${ }^{65,66}$, W. P. Chen ${ }^{67}$, C. Diltz ${ }^{62}$,

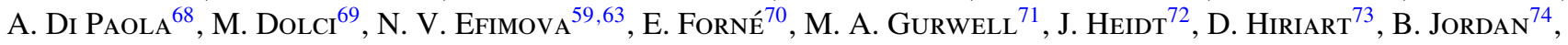
G. Kimeridze $^{75}$, T. S. Konstantinova ${ }^{63}$, E. N. Kopatskaya ${ }^{63}$, E. Koptelova ${ }^{67,76}$, O. M. Kurtanidze ${ }^{75}$, A. LÄhteenmäKi ${ }^{77}$, E. G. Larionova ${ }^{63}$, L. V. Larionova ${ }^{63}$, V. M. Larionov ${ }^{78,59,63}$, P. Leto $^{64}$, E. LindFors ${ }^{61}$, H. C. Lin ${ }^{67}$, D. A. Morozova ${ }^{63}$, M. G. Nikolashitili ${ }^{75}$, K. Nilsson ${ }^{79}$, M. Oksman ${ }^{77}$, P. RoustaZadeh ${ }^{62}$, A. Sievers ${ }^{80}$, L. A. Sigua ${ }^{75}$, A. Sillanpä̈̈ ${ }^{61}$, T. Takahashi ${ }^{44}$, L. O. TaKalo ${ }^{61}$, M. Tornikoski ${ }^{77}$, C. Trigilio ${ }^{64}$, I. S. Troitsky ${ }^{63}$, G. Umana ${ }^{64}$ (THE GASP-WEBT CONSORTIUM) AND E. Angelakis ${ }^{24}$, T. P. Krichbaum ${ }^{24}$, I. Nestoras ${ }^{24}$, D. Riquelme
(F-GAMMA) AND M. KRIPS ${ }^{81}$, S. TRIPPE ${ }^{82}$ (IRAM-PDBI) AND

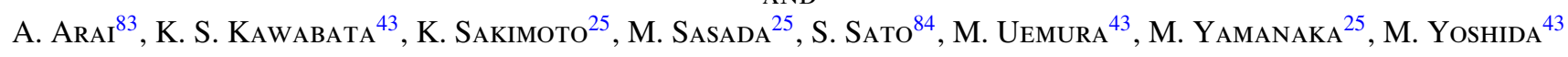
(Kanata) AND T. BELLONI ${ }^{85}$, G. TAGLIAFERRI ${ }^{85}$ (RXTE) AND E. W. BONNING ${ }^{86}$, J. ISLER ${ }^{86}$, C. M. URRY ${ }^{86}$ (SMARTS) AND E. Hoversten ${ }^{87}$, A. Falcone ${ }^{87}$, C. Pagani ${ }^{88}$, M. StroH $^{87}$ (Swift-XRT)

${ }^{1}$ Deutsches Elektronen Synchrotron DESY, D-15738 Zeuthen, Germany

${ }^{2}$ W. W. Hansen Experimental Physics Laboratory, Kavli Institute for Particle Astrophysics and Cosmology, Department of Physics and SLAC National Accelerator Laboratory, Stanford University, Stanford, CA 94305, USA; eduardo@slac.stanford.edu, madejski@ slac.stanford.edu ${ }^{3}$ Laboratoire AIM, CEA-IRFU/CNRS/Université Paris Diderot, Service d'Astrophysique, CEA Saclay, 91191 Gif sur Yvette, France ${ }^{4}$ Istituto Nazionale di Fisica Nucleare, Sezione di Trieste, I-34127 Trieste, Italy ${ }^{5}$ Dipartimento di Fisica, Università di Trieste, I-34127 Trieste, Italy

${ }^{6}$ Istituto Nazionale di Fisica Nucleare, Sezione di Padova, I-35131 Padova, Italy

${ }^{7}$ Dipartimento di Fisica "G. Galilei," Università di Padova, I-35131 Padova, Italy

${ }^{8}$ Istituto Nazionale di Fisica Nucleare, Sezione di Pisa, I-56127 Pisa, Italy 


\author{
${ }^{9}$ Istituto Nazionale di Fisica Nucleare, Sezione di Perugia, I-06123 Perugia, Italy \\ ${ }^{10}$ Dipartimento di Fisica, Università degli Studi di Perugia, I-06123 Perugia, Italy \\ ${ }^{11}$ Dipartimento di Fisica “M. Merlin” dell’Università e del Politecnico di Bari, I-70126 Bari, Italy \\ ${ }^{12}$ Istituto Nazionale di Fisica Nucleare, Sezione di Bari, 70126 Bari, Italy; fabio.gargano@ba.infn.it, silvia.raino@ba.infn.it \\ ${ }^{13}$ Laboratoire Leprince-Ringuet, École polytechnique, CNRS/IN2P3, Palaiseau, France \\ ${ }^{14}$ Institut de Ciències de l'Espai (IEEE-CSIC), Campus UAB, 08193 Barcelona, Spain \\ ${ }^{15}$ INAF-Istituto di Astrofisica Spaziale e Fisica Cosmica, I-20133 Milano, Italy \\ ${ }^{16}$ Agenzia Spaziale Italiana (ASI) Science Data Center, I-00044 Frascati (Roma), Italy \\ ${ }^{17}$ Artep Inc., 2922 Excelsior Springs Court, Ellicott City, MD 21042, USA \\ ${ }^{18}$ ASI Science Data Center, I-00044 Frascati (Roma), Italy \\ ${ }^{19}$ Laboratoire Univers et Particules de Montpellier, Université Montpellier 2, CNRS/IN2P3, Montpellier, France \\ ${ }^{20}$ IASF Palermo, 90146 Palermo, Italy \\ ${ }^{21}$ INAF-Istituto di Astrofisica Spaziale e Fisica Cosmica, I-00133 Roma, Italy \\ ${ }^{22}$ Space Science Division, Naval Research Laboratory, Washington, DC 20375-5352, USA \\ ${ }^{23}$ NASA Goddard Space Flight Center, Greenbelt, MD 20771, USA \\ ${ }^{24}$ Max-Planck-Institut für Radioastronomie, Auf dem Hügel 69, 53121 Bonn, Germany \\ ${ }^{25}$ Department of Physical Sciences, Hiroshima University, Higashi-Hiroshima, Hiroshima 739-8526, Japan \\ ${ }^{26}$ INAF Istituto di Radioastronomia, 40129 Bologna, Italy \\ ${ }^{27}$ Center for Space Plasma and Aeronomic Research (CSPAR), University of Alabama in Huntsville, Huntsville, AL 35899, USA \\ ${ }^{28}$ Department of Astronomy, Graduate School of Science, Kyoto University, Sakyo-ku, Kyoto 606-8502, Japan \\ ${ }^{29}$ Department of Physics, Center for Cosmology and Astro-Particle Physics, The Ohio State University, Columbus, OH 43210, USA \\ ${ }^{30}$ Science Institute, University of Iceland, IS-107 Reykjavik, Iceland \\ ${ }^{31}$ College of Science, Ibaraki University, 2-1-1 Bunkyo, Mito 310-8512, Japan \\ ${ }^{32}$ Research Institute for Science and Engineering, Waseda University, 3-4-1, Okubo, Shinjuku, Tokyo 169-8555, Japan \\ ${ }^{33}$ CNRS, IRAP, F-31028 Toulouse cedex 4, France \\ ${ }^{34}$ GAHEC, Université de Toulouse, UPS-OMP, IRAP, Toulouse, France \\ ${ }^{35}$ Department of Physics, Stockholm University, AlbaNova, SE-106 91 Stockholm, Sweden \\ 36 The Oskar Klein Centre for Cosmoparticle Physics, AlbaNova, SE-106 91 Stockholm, Sweden \\ ${ }^{37}$ Department of Astronomy, Stockholm University, SE-106 91 Stockholm, Sweden \\ ${ }^{38}$ Yukawa Institute for Theoretical Physics, Kyoto University, Kitashirakawa Oiwake-cho, Sakyo-ku, Kyoto 606-8502, Japan \\ ${ }^{39}$ CNRS/IN2p3, Centre d'Études Nucléaires de Bordeaux Gradignan, Université Bordeaux 1, 33175 Gradignan, France \\ ${ }^{40}$ Department of Physics and Department of Astronomy, University of Maryland, College Park, MD 20742, USA \\ ${ }^{41}$ Istituto Nazionale di Fisica Nucleare, Sezione di Roma "Tor Vergata," I-00133 Roma, Italy \\ ${ }^{42}$ Department of Physics, Boise State University, Boise, ID 83725, USA \\ ${ }^{43}$ Hiroshima Astrophysical Science Center, Hiroshima University, Higashi-Hiroshima, Hiroshima 739-8526, Japan \\ ${ }^{44}$ Institute of Space and Astronautical Science, JAXA, 3-1-1 Yoshinodai, Chuo-ku, Sagamihara, Kanagawa 252-5210, Japan \\ ${ }^{45}$ Max-Planck Institut für extraterrestrische Physik, 85748 Garching, Germany \\ ${ }^{46}$ Max-Planck-Institut für Physik, D-80805 München, Germany \\ ${ }^{47}$ Santa Cruz Institute for Particle Physics, Department of Physics and Department of Astronomy and Astrophysics, \\ University of California at Santa Cruz, Santa Cruz, CA 95064, USA \\ ${ }^{48}$ Cahill Center for Astronomy and Astrophysics, California Institute of Technology, Pasadena, CA 91125, USA \\ 49 Institut für Astro- und Teilchenphysik and Institut für Theoretische Physik, Leopold-Franzens-Universität Innsbruck, A-6020 Innsbruck, Austria \\ ${ }^{50}$ Department of Physics, California Polytechnic State University, San Luis Obispo, CA 93401, USA; 1reyes04@ calpoly.edu \\ ${ }^{51}$ NYCB Real-Time Computing Inc., Lattingtown, NY 11560-1025, USA \\ ${ }^{52}$ Institució Catalana de Recerca i Estudis Avançats (ICREA), Barcelona, Spain \\ ${ }^{53}$ Consorzio Interuniversitario per la Fisica Spaziale (CIFS), I-10133 Torino, Italy \\ ${ }^{54}$ Dipartimento di Fisica, Università di Roma “Tor Vergata," I-00133 Roma, Italy \\ ${ }^{55}$ Nicolaus Copernicus Astronomical Center, 00-716 Warsaw, Poland; knalew@ colorado.edu, sikora@ @amk.edu.pl \\ ${ }^{56}$ University of Colorado, 440 UCB, Boulder, CO 80309, USA \\ ${ }^{57}$ INAF, Osservatorio Astronomico di Torino, I-10025 Pino Torinese (TO), Italy \\ ${ }^{58}$ Department of Astronomy, University of Michigan, Ann Arbor, MI 48109-1042, USA \\ ${ }^{59}$ Pulkovo Observatory, 196140 St. Petersburg, Russia \\ ${ }^{60}$ Instituto de Astronomía, Universidad Nacional Autónoma de México, México, D. F., Mexico \\ ${ }^{61}$ Tuorla Observatory, University of Turku, FI-21500 Piikkiö, Finland \\ ${ }^{62}$ Department of Physics and Astronomy, Ohio University, Athens, OH 45701, USA \\ ${ }^{63}$ Astronomical Institute, St. Petersburg State University, St. Petersburg, Russia \\ ${ }^{64}$ Osservatorio Astrofisico di Catania, 95123 Catania, Italy \\ ${ }^{65}$ EPT Observatories, Tijarafe, La Palma, Spain \\ ${ }^{66}$ INAF, TNG Fundacion Galileo Galilei, La Palma, Spain \\ ${ }^{67}$ Graduate Institute of Astronomy, National Central University, Jhongli 32054, Taiwan \\ ${ }^{68}$ Osservatorio Astronomico di Roma, I-00040 Monte Porzio Catone (Roma), Italy \\ ${ }^{69}$ Osservatorio Astronomico di Collurania "Vincenzo Cerruli," 64100 Teramo, Italy \\ ${ }^{70}$ Agrupació Astronòmica de Sabadell, 08206 Sabadell, Spain \\ ${ }^{71}$ Harvard-Smithsonian Center for Astrophysics, Cambridge, MA 02138, USA \\ ${ }^{72}$ Landessternwarte, Universität Heidelberg, Königstuhl, D 69117 Heidelberg, Germany \\ ${ }^{73}$ Instituto de Astronomía, Universidad Nacional Autónoma de México, Ensenada, B. C., Mexico \\ ${ }^{74}$ School of Cosmic Physics, Dublin Institute for Advanced Studies, Dublin, 2, Ireland \\ ${ }^{75}$ Abastumani Observatory, Mt. Kanobili, 0301 Abastumani, Georgia \\ ${ }^{76}$ Department of Physics, National Taiwan University, 106 Taipei, Taiwan \\ ${ }_{77}^{77}$ Metsähovi Radio Observatory, Aalto University, FIN-02540 Kylmala, Finland \\ ${ }^{78}$ Isaac Newton Institute of Chile, St. Petersburg Branch, St. Petersburg, Russia \\ ${ }^{79}$ Finnish Centre for Astronomy with ESO (FINCA), University of Turku, FI-21500 Piikiiö, Finland \\ ${ }^{80}$ Institut de Radio Astronomie Millimètrique, Avenida, Divina Pastora 7, Local 20, 18012 Granada, Spain \\ ${ }^{81}$ Institut de Radioastronomie Millimétrique, Domaine Universitaire, 38406 Saint Martin d'Hères, France \\ 82 Department of Physics and Astronomy, Seoul National University, Seoul, 151-742, Korea
}




$$
\begin{gathered}
{ }^{83} \text { Department of Physics, Graduate School of Science, Kyoto University, Kyoto, Japan } \\
{ }^{84} \text { Department of Physics and Astrophysics, Nagoya University, Chikusa-ku Nagoya 464-8602, Japan } \\
\text { 85 INAF Osservatorio Astronomico di Brera, I-23807 Merate, Italy } \\
{ }^{86} \text { Department of Astronomy, Department of Physics and Yale Center for Astronomy and Astrophysics, Yale University, New Haven, CT 06520-8120, USA } \\
87 \text { Department of Astronomy and Astrophysics, Pennsylvania State University, University Park, PA 16802, USA } \\
{ }^{88} \text { Department of Physics and Astronomy, University of Leicester, Leicester LE1 7RH, UK } \\
\text { Received 2011 October 20; accepted 2012 March 23; published 2012 May 17 }
\end{gathered}
$$

\begin{abstract}
The blazar AO $0235+164(z=0.94)$ has been one of the most active objects observed by Fermi Large Area Telescope (LAT) since its launch in Summer 2008. In addition to the continuous coverage by Fermi, contemporaneous observations were carried out from the radio to $\gamma$-ray bands between 2008 September and 2009 February. In this paper, we summarize the rich multi-wavelength data collected during the campaign (including F-GAMMA, GASPWEBT, Kanata, OVRO, RXTE, SMARTS, Swift, and other instruments), examine the cross-correlation between the light curves measured in the different energy bands, and interpret the resulting spectral energy distributions in the context of well-known blazar emission models. We find that the $\gamma$-ray activity is well correlated with a series of near-IR/optical flares, accompanied by an increase in the optical polarization degree. On the other hand, the X-ray light curve shows a distinct 20 day high state of unusually soft spectrum, which does not match the extrapolation of the optical/UV synchrotron spectrum. We tentatively interpret this feature as the bulk Compton emission by cold electrons contained in the jet, which requires an accretion disk corona with an effective covering factor of $19 \%$ at a distance of $100 R_{\mathrm{g}}$. We model the broadband spectra with a leptonic model with external radiation dominated by the infrared emission from the dusty torus.
\end{abstract}

Key words: BL Lacertae objects: individual (AO 0235+164) - galaxies: active - galaxies: jets - gamma rays: galaxies - radiation mechanisms: non-thermal

Online-only material: color figures

\section{INTRODUCTION}

Blazars are a class of active galactic nuclei (AGNs) characterized by high flux variability at all wavelengths and compact (milliarcsecond scale) radio emission of extreme brightness temperatures, often exceeding the Compton limit (Urry 1999). Their radio spectra are generally well described by a power-law shape, with a "flat" spectral index $\alpha<0.5$ (where the flux density $\left.F_{v} \propto v^{-\alpha}\right)$. Multi-epoch Very Long Baseline Interferometry (VLBI) observations often show superluminal expansion, and the radio and optical emission is usually highly polarized. These general properties are well described as arising in a relativistic jet pointing close to our line of sight (Blandford \& Rees 1978). The jet, presumably deriving its power from accretion onto a supermassive, rotating black hole $(\mathrm{BH})$ surrounded by an accretion disk, contains ultrarelativistic electrons (with particle Lorentz factors $\gamma_{\mathrm{el}}$ reaching $10^{3}-10^{5}$, depending on the object). These relativistic electrons produce soft photons from radio up to UV (or in some cases, soft X-rays) through synchrotron emission, and high-energy photons up to $\mathrm{TeV}$ energies, via the inverse Compton process which involves scattering of synchrotron photons (the SSC scenario), as well as scattering of externally produced soft photons (the External Radiation Compton, ERC, scenario). A contribution to the high-energy radiation can also be provided by synchrotron radiation of pair cascades powered by hadronic processes and by synchrotron emission of ultra-high-energy protons and muons (see reviews of radiative models of blazars by Sikora \& Madejski 2001; Levinson 2006; Böttcher 2007). Noting difficulties of hadronic models to explain the spectra of luminous blazars (Sikora et al. 2009; Sikora 2011), we investigate in this paper only leptonic models, i.e., the models which involve production of radiation by directly accelerated electrons. Densely sampled, simultaneous

\footnotetext{
${ }^{89}$ Resident at Naval Research Laboratory, Washington, DC 20375, USA.

90 NASA Postdoctoral Program Fellow, USA.
}

monitoring observations throughout the entire electromagnetic spectrum from the radio to $\gamma$-ray bands can provide important constraints on such models.

When emission lines are absent or weak, with an equivalent width (EW) less than $5 \AA$ in the rest frame (see, e.g., Stickel et al. 1991), a blazar is classified as a BL Lac object; otherwise, it belongs to the class of flat-spectrum radio quasars (FSRQs). While in a majority of BL Lac objects-especially in those with the $v F_{v}$ spectral energy distribution (SED) peaking in the far UV-to-X-ray range (the so-called HSP or high-synchrotronpeaked BL Lac objects) - detection of emission lines is rare, and if detected, the lines are extremely weak (for recent measurements, see, e.g., Stocke et al. 2011), in the objects where the SED peaks in the infrared or optical range (the so-called LSP or low-synchrotron-peaked BL Lac objects), easily discernible emission lines have been detected often. When detected, such lines provide a measurement of redshift, but also yield crucial information about the details of accretion in the central source. In some cases such as AO 0235+164 (Raiteri et al. 2007), discussed in this paper, and even BL Lacertae (Vermeulen et al. 1995; Corbett et al. 2000), the prototype of the BL Lac class, the $\mathrm{EW}$ of emission lines can vary from one observational epoch to another. This is primarily due to the large-amplitude variability of the nonthermal continuum, which becomes brighter or fainter with respect to the presumably less-variable emission lines. Regardless, the detailed properties of the emission lines are crucial in establishing the radiative environment encountered by the jet emerging from the nucleus, and thus are indispensable in establishing the most likely source of seed-photon population for inverse Compton scattering. While the most compelling scenario has the internal jet photons dominating this population in the HSP sub-class, and the external photons (from emission-line region, or disk photons rescattered by the medium confining the lines) in FSRQs, the situation with LSP BL Lac objects is unclear. 
Studies of an LSP blazar AO $0235+164$ provide an exceptional opportunity to answer this question. It is one of the original BL Lac objects in the Stein et al. (1976) compilation, discovered via optical identification of a variable radio source by Spinrad \& Smith (1975). Early observations - as well as the inspection of historical plates - revealed that optical variability can range over 5 mag (Rieke et al. 1976), motivating monitoring observations over a wide range of frequencies since its discovery. The redshift $z_{\mathrm{em}}=0.94$ has been inferred from weak optical emission lines by Cohen et al. (1987), but even earlier optical spectroscopy revealed two absorption line systems, one at $z_{\mathrm{ab} 1}=0.524$, and another, weaker one at $z_{\mathrm{ab} 2}=0.852$ discovered by Burbidge et al. (1976) and by Rieke et al. (1976). The intervening $z_{\mathrm{ab} 1}=0.524$ system has also been detected in absorption in the radio, via the redshifted hydrogen $21 \mathrm{~cm}$ line by Wolfe \& Wills (1977) and Roberts (1976), but also as an Ly $\alpha$ absorber, revealing damped Ly $\alpha$ properties (Snijders 1982) and implying a considerable absorption in other bands. Detailed studies of that absorbing system by Junkkarinen et al. (2004) allow accurate corrections to be applied to the observed optical spectra in order to determine reliably the intrinsic spectrum of the blazar. Likewise, since the environment in the field of AO $0235+164$ is complex and includes several possibly interacting foreground galaxies at $z_{\mathrm{ab} 1}=0.524$ as well as the system at $z_{\mathrm{ab} 2}=0.852$, the emission in the optical-UV band (and to a much lesser degree, in the soft X-ray band) may be contaminated. One galaxy, probably a normal spiral, is 1.3 arcsec east, while another object, about 2 arcsec to the south, is known to be an AGN and could affect the flux of AO 0235+164 when it is very faint, especially in the bluer part of the spectrum (Raiteri et al. 2005).

Historical data for this source are abundant. Radio observations were performed by many instruments, starting from about $100 \mathrm{MHz}$ up to $300 \mathrm{GHz}$, and including multi-epoch VLBI studies (Jorstad et al. 2001). Space and ground-based infrared data are available from submillimeter (sub-mm; far-IR) down to micron wavelengths (near-IR); optical bands, UBVRI, have been extensively monitored by many telescopes around the world. AO $0235+164$ has also been detected in the highenergy band by essentially all soft X-ray observatories including Einstein (Worral \& Wilkes 1990), EXOSAT (Ghosh \& Soundararajaperumal 1995), ROSAT (Madejski et al. 1996; Comastri et al. 1997), ASCA (Madejski et al. 1996; Junkkarinen et al. 2004), Beppo-SAX (Padovani et al. 2004), RXTE (Webb et al. 2000), and XMM-Newton (Raiteri et al. 2008). This source has also been identified as a powerful and strongly variable $\gamma$-ray emitter via observations by EGRET on board the Compton Gamma-Ray Observatory (CGRO) in the high $\gamma$-ray energy range from $30 \mathrm{MeV}$ to $20 \mathrm{GeV}$, with six pointings between 1992 and 1997 providing two detections (Hunter et al. 1993; Madejski et al. 1996) and four upper limits. The mid-energy $\gamma$-ray emission was probed by COMPTEL during CGRO Cycle 4 (1994-1995), yielding only upper limits for the flux in the interval of $0.75-30 \mathrm{MeV}$. These numerous multiwavelength observations show that AO $0235+164$ is characterized by extreme variability on long (month-years) and short (intraday) timescales over a wide range of the electromagnetic spectrum.

The study of blazars, of their broadband spectra and of their complex variability, has been greatly enriched since the start of scientific observations with the Fermi Large Area Telescope (LAT) in 2008 August (Atwood et al. 2009) thanks to its high sensitivity and essentially uninterrupted observations afforded by the survey mode. Such new and sensitive $\gamma$-ray observations motivated many multi-band campaigns, often conducted with dedicated facilities, and AO $0235+164$ was (and continues to be) one of the well-sampled targets. This paper presents the results of the LAT monitoring of AO $0235+164$, as reported in Section 2. The description of multi-wavelength observations conducted between 2008 August and 2009 February when the source showed strong activity in $\gamma$-rays as well as in radio through optical and X-ray bands (Corbel \& Reyes 2008; Foschini et al. 2008) follows in Section 3. The analysis of those data, including the discussion of the temporal profiles measured in various bands and the connection to the $\gamma$-ray activity, is reported in Section 4. A significant part of these data have been independently analyzed by Agudo et al. (2011b). In Section 5, we present the overall SED and its temporal behavior, and discuss the implications of the data on the modeling of emission processes and the structure of the jet in AO 0235+164: there, we argue that while the EW of emission lines in this object might suggest a classification as a BL Lac object, the isotropic luminosity inferred from the data indicates it is a quasar. In Section 6, we show models of the broadband emission in the context of synchrotron + Compton models. Our consideration of the broadband SED suggests that the most likely mechanism for $\gamma$-ray emission is the Comptonization of circumnuclear IR radiation from dust, commonly present in quasars. This is a different scenario from the one proposed by Agudo et al. (2011b), who argued for the synchrotron self-Compton process. We discuss these two approaches in Section 7. We conclude with a summary of our results in Section 8.

\section{FERMI-LAT OBSERVATIONS AND DATA ANALYSIS}

The LAT, the primary instrument on board the Fermi $\gamma$-ray observatory, is an electron-positron pair conversion telescope sensitive to $\gamma$-rays of energies from $20 \mathrm{MeV}$ to $>300 \mathrm{GeV}$. The LAT consists of a high-resolution silicon microstrip tracker, a CsI hodoscopic electromagnetic calorimeter, and an anticoincidence detector for the identification of charged particles' background. The full description of the instrument and its performance can be found in Atwood et al. (2009). The large field of view ( $2.4 \mathrm{sr})$ allows the LAT to observe the full sky in survey mode every $3 \mathrm{hr}$. The LAT point-spread function (PSF) strongly depends on both the energy and the conversion point in the tracker, but less so on the incidence angle. For $1 \mathrm{GeV}$ normalincidence conversions in the upper section of the tracker, the PSF $68 \%$ containment radius is 0.8 .

The Fermi-LAT data of AO $0235+164$ presented here were obtained in the time period between 2008 August and 2009 February when AO $0235+164$ entered a bright high $\gamma$-ray state, and immediately after, dropped to lower states. The data have been analyzed by using the standard Fermi-LAT software package. ${ }^{91}$ The Pass 6 Diffuse event class and P6_V3_DIFFUSE instrument response functions (Atwood et al. 2009) were used in our analysis. We selected events within a $15^{\circ}$ region of interest centered on the source position, having energy greater than $100 \mathrm{MeV}$. The data have been analyzed using the Science Tools software package (version v9r16). In order to avoid background contamination from the bright Earth limb, time intervals when the Earth entered the LAT Field of View were excluded from the data set. In addition, events with zenith angles larger than $105^{\circ}$ with respect to the Earth reference frame (Abdo et al. 2009) were excluded from the analysis. The data were analyzed with an

\footnotetext{
91 http://fermi.gsfc.nasa.gov/ssc/data/analysis/documentation/Cicerone/
} 
unbinned maximum likelihood technique described by Mattox et al. (1996) using the analysis software (gtlike) developed by the LAT team. ${ }^{92}$

Accurate spectral and flux measurements require a reliable accounting for the diffuse foreground due to the Galactic interstellar emission, as well as the extragalactic diffuse $\gamma$-ray emission, the residual cosmic-ray background, and contamination from nearby sources. The fitting procedure simultaneously fits for the parameters of the source of interest as well as of nearby $\gamma$-ray sources and the diffuse backgrounds, which in turn have been modeled using gll_iem_v02 for the Galactic diffuse emission and isotropic_iem_v02 for the extragalactic isotropic emission models. ${ }^{93}$

The sources surrounding AO 0235+164 were modeled using a power-law function:

$$
\frac{d N}{d E}=\frac{N(1-\Gamma) E^{-\Gamma}}{E_{\max }^{1-\Gamma}-E_{\min }^{1-\Gamma}},
$$

where $N$ is the normalization factor and $\Gamma$ the photon index. In the fitting procedure, all sources within $10^{\circ}$ were included in the model with the normalization factor $N$ free, while the sources located between $10^{\circ}$ and $20^{\circ}$ had all the model parameters fixed to the 1FGL catalog values (Abdo et al. 2010a).

The plots in subsequent sections show only statistical errors for the fit parameters. Systematic errors arise mainly from uncertainties on the LAT effective area, which is derived from the on-orbit estimations. These errors could be as large as $10 \%$ below $0.1 \mathrm{GeV},<5 \%$ near $1 \mathrm{GeV}$, and $20 \%$ above $10 \mathrm{GeV}$.

\section{1. $\gamma$-Ray Light Curve}

The light curve of AO $0235+164$ in the Fermi-LAT energy range has been assembled using three day long time bins and covers the first six months of data taken from 2008 August 4 to 2009 February 4, when the source was in a high state and a large set of multi-wavelength observations were available. The light curve is obtained by applying the gtlike fit across the overall energy range considered, from $100 \mathrm{MeV}$ to $100 \mathrm{GeV}$, in each of the selected time bins. For each time interval, the flux and the photon index of AO $0235+164$ are determined using the maximum likelihood algorithm implemented in gtlike, following the procedure outlined in the previous section. The data are modeled with a power-law function with both the normalization factor and photon index left free in the likelihood fit.

The six month $\gamma$-ray light curve is reported in Figure 1 together with the photon index resulting from the likelihood fit in each time bin. The trend in the entire energy range from $100 \mathrm{MeV}$ to $100 \mathrm{GeV}$ shows a clear high-state period followed by a final, narrow, high-flux peak.

The temporal behavior of the source in $\gamma$-rays was also studied in two separate energy ranges, from $100 \mathrm{MeV}$ to $1 \mathrm{GeV}$ and from $1 \mathrm{GeV}$ to $100 \mathrm{GeV}$, and the hardness ratio among the two bands has been determined. The analysis follows the same procedure described above to determine the overall light curve and the results are shown in the three bottom panels of Figure 1.

The arrows in the light curves represent $95 \%$ upper limits, which are calculated for data points with a test statistic (TS) ${ }^{94}$

\footnotetext{
92 http://fermi.gsfc.nasa.gov/ssc/data/analysis/documentation/Cicerone/ Cicerone_Likelihood

93 http://fermi.gsfc.nasa.gov/ssc/data/access/lat/BackgroundModels.html 94 The test statistics is defined as TS $=-2 \times\left(\log \left(L_{1}\right)-\log \left(L_{0}\right)\right)$ with $L_{0}$ the likelihood of the Null-hypothesis model as compared to the likelihood of a competitive model, $L_{1}$; see Mattox et al. (1996).
}

lower than 10 (which corresponds to a significance somewhat higher than $3 \sigma$ ) or with a value of the ratio between flux error and flux $\left(F_{\mathrm{err}}(E) / F(E)\right) \geqslant 0.5$ in order to obtain meaningful data points.

The results show that both the low- and high-energy profiles follow the same trend. Nevertheless, it is interesting to underline that the narrow peak at the end of the high-state period is mainly due to an enhanced low-energy flux. The ratio among the two fluxes also shows a value higher than the average in the same time interval.

\section{2. $\gamma$-Ray Spectral Analysis}

The unbinned gtlike analysis has been applied to produce the $\gamma$-ray energy spectra shown in Figure 2. There, we divided the full energy range from $100 \mathrm{MeV}$ to $100 \mathrm{GeV}$ into two equal logarithmically spaced bins per decade. In each energy bin, a TS value greater than 10 and a ratio between flux error and flux lower than 0.5 was required to quote a flux in that band, otherwise a $95 \%$ upper limit was given.

The standard gtlike tool was applied in each energy bin, modeling all the point sources in the region with a simple power-law spectrum with photon index fixed to two. The normalization parameters of all point-like sources within $10^{\circ}$ were left as free parameters in the fitting procedure, while the diffuse background components were modeled as described above in Section 2.1. Two time intervals were selected for the $\gamma$-ray spectral analysis: the first corresponding to the X-ray flare interval (MJD 54750-54770), the second associated with the subsequent low $\gamma$-ray state (MJD 54780-54840). In those time intervals, both power-law and broken-power-law functions provide a good fit of the spectral data. In Table 1, we show the results of the broken-power-law fit, since it provides a better fit to the high-energy spectrum of the source, from $100 \mathrm{MeV}$ to $100 \mathrm{GeV}$, than a simple power law on the larger time intervals, as already studied in detail by Abdo et al. (2010b):

$$
\frac{d N}{d E}=N_{0} \times \begin{cases}\left(E / E_{b}\right)^{-\Gamma_{1}} ; & \text { if } E<E_{b} \\ \left(E / E_{b}\right)^{-\Gamma_{2}} ; & \text { other wise }\end{cases}
$$

In both time intervals, the $\Gamma_{1}$ index remains stable, while $\Gamma_{2}$ increases showing a softening of the high-energy part of the spectrum when the source is in a fainter state, when also an increase in the break energy is observed. As also can be seen in Figure 1, the high $\gamma$-ray state around MJD 54760 is essentially due to the low-energy photons $(<1 \mathrm{GeV})$ and the spectra in Figure 2 show that the relative difference between the $E^{2}$ Flux values above $1 \mathrm{GeV}$ and below $1 \mathrm{GeV}$ is higher in the time interval around the flare than during the low $\gamma$-ray state.

\section{MULTI-WAVELENGTH OBSERVATIONS AND DATA ANALYSIS}

The multi-wavelength campaign conducted on AO $0235+164$ in 2008-2009 saw a wide international participation. Table 2 reports the list of participating observatories, the energy bands, the period of observation, and the number of collected data points.

\subsection{Effect of Intervening Material in the Line of Sight on the Optical, UV and X-Ray Data}

Conversion of the observed optical magnitudes into the intrinsic flux densities requires a special care, because the source emission is absorbed not only in our Galaxy, but 


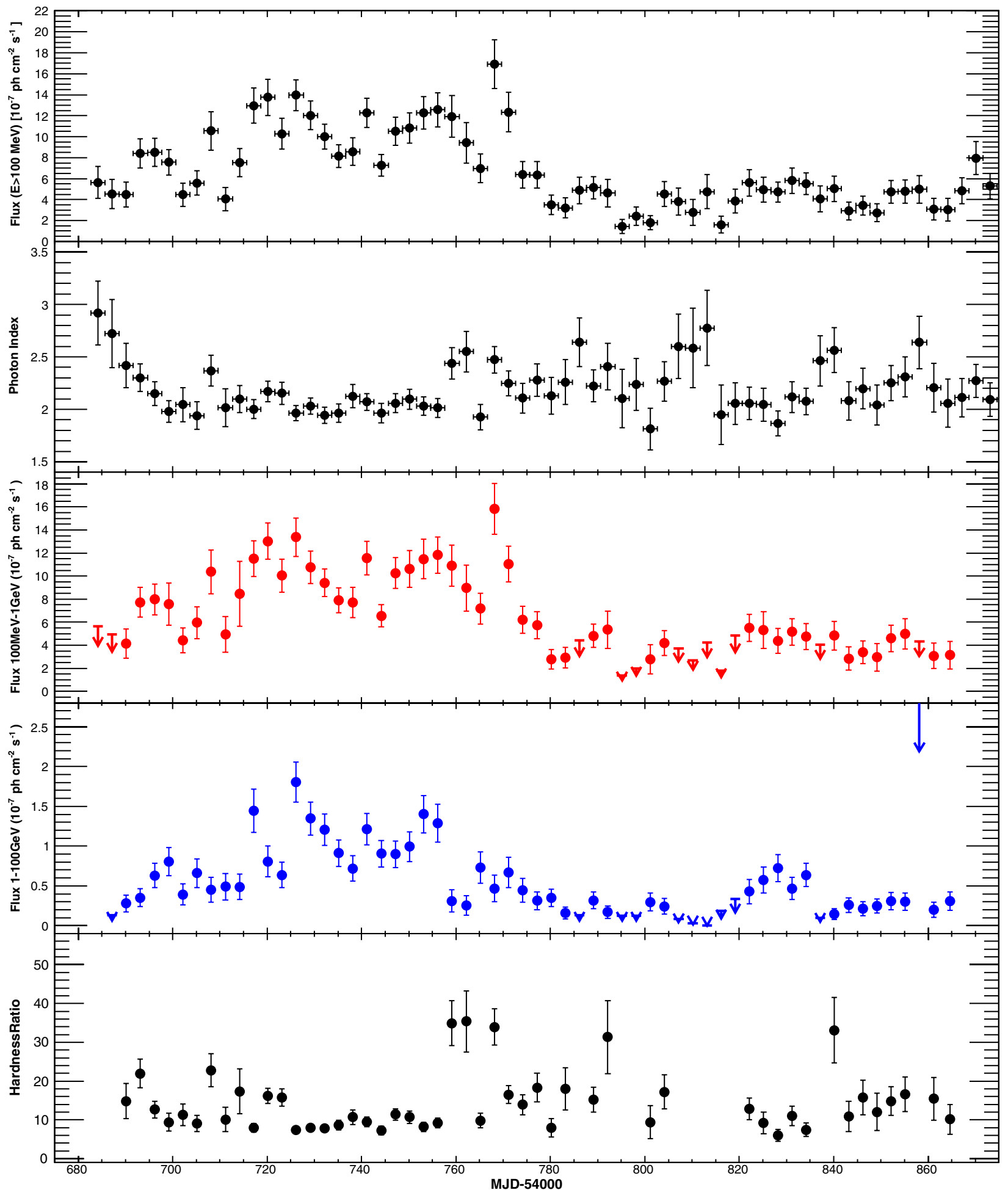

Figure 1. Fermi-LAT light curve from 2008 August 4 to 2009 February 4 in three day time intervals. The first panel from the top shows the flux, in the energy range from $100 \mathrm{MeV}$ to $100 \mathrm{GeV}$, derived from the gtlike fit in the three day time intervals, assuming a simple power-law spectrum. The second panel shows the photon index $\Gamma$ in the same energy range from $100 \mathrm{MeV}$ to $100 \mathrm{GeV}$. The third panel shows the light curve evaluated in the energy range from $100 \mathrm{MeV}$ to $1 \mathrm{GeV}$. The fourth

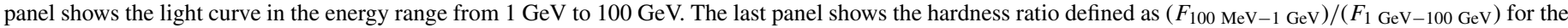
data points having a TS $>10$ and $F_{\mathrm{err}}(E) / F(E)>0.5$ in both energy ranges. The hardness ratio is not evaluated if either of the two fluxes is an upper limit.

(A color version of this figure is available in the online journal.)

Table 1

Results of the gtlike Fit of the $\gamma$-ray Spectrum During the High and Low States

\begin{tabular}{lcccc}
\hline \hline $\begin{array}{l}\text { Time Interval } \\
\text { MJD }\end{array}$ & $\begin{array}{c}\text { Flux } \\
\left(10^{-7} \text { photons cm }{ }^{-2} \mathrm{~s}^{-1}\right)\end{array}$ & $\Gamma_{1}$ & $\Gamma_{2}$ & $\begin{array}{c}\text { Break Energy } \\
(\mathrm{GeV})\end{array}$ \\
\hline $54750-54770$ & $11.05 \pm 1.48$ & $2.12 \pm 0.16$ & $2.37 \pm 0.32$ & $2.6 \pm 0.8$ \\
$54780-54840$ & $3.42 \pm 0.65$ & $2.07 \pm 0.17$ & $2.77 \pm 0.32$ & $3.8 \pm 1.2$ \\
\hline
\end{tabular}




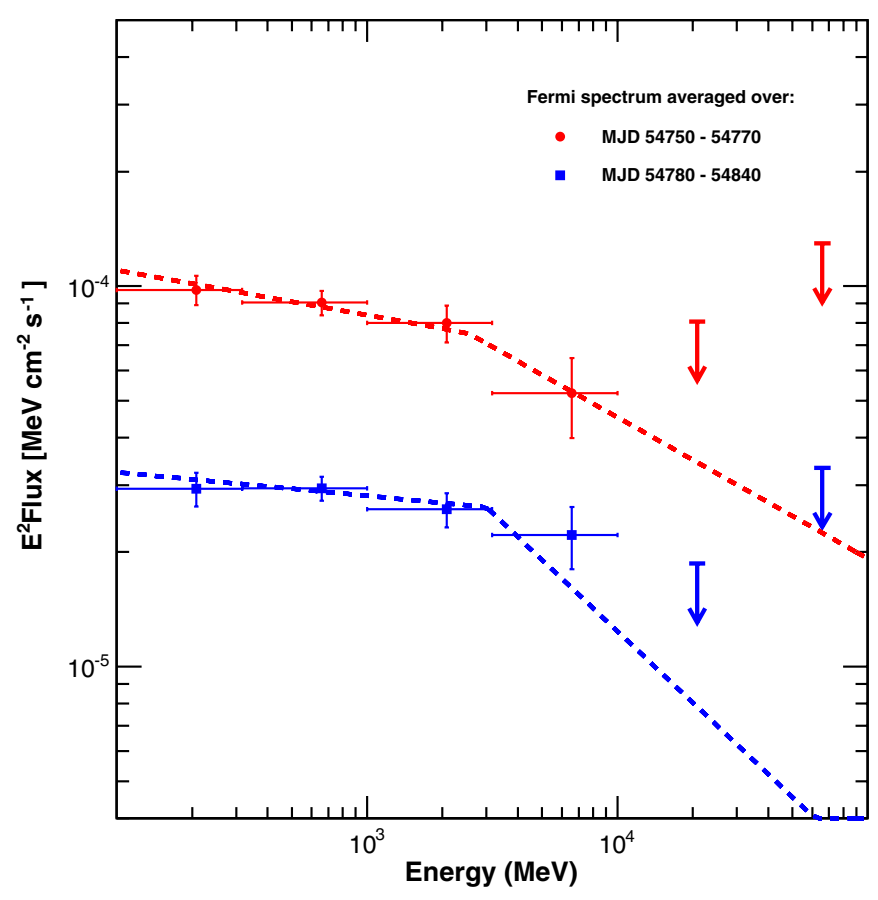

Figure 2. Fermi-LAT energy spectrum evaluated in different time intervals corresponding to the X-ray flare (red circles) from MJD 54750-54770 and $\gamma$-ray low state (blue squares) from MJD 54780-54840.

(A color version of this figure is available in the online journal.)

also by the elliptical galaxy in the line of sight at redshift $z=0.524$, as outlined in the Introduction. Junkkarinen et al. (2004) tried several different extinction models for AO 0235+164, concluding that the best fit to their Hubble Space Telescope/STIS data is obtained by using models of Cardelli et al. (1989) with $R_{\mathrm{V}}=3.1$ and $E_{\mathrm{B}-\mathrm{V}}=0.154$ for the Galaxy, and $R_{\mathrm{V}}=2.51$ and $E_{\mathrm{B}-\mathrm{V}}=0.227$ for the $z=0.524$ system. This model accurately reproduces the $2175 \AA$ absorption feature produced by the $z=0.524$ galaxy, but the far-UV end of their spectrum indicated a sharp hardening. Raiteri et al. (2005) proposed that this far-UV hardening is real and that it marks the onset of a new spectral component. However, the fact that the shape of this feature does not change with the overall optical/UV luminosity indicates that the whole optical/UV spectrum is produced by a single synchrotron component, the intrinsic shape of which must be close to a power law. The far-UV hardening is most likely an artifact of overestimated extinction from the dust in the $z=0.524$ galaxy. We modify the best-fit extinction model of Junkkarinen et al. (2004) by replacing the Cardelli et al. (1989) model for the $z=0.524$ galaxy with an analytical model of Pei (1992). In the first step, we modify the "Milky Way" model with parameters listed in Table 4 of Pei (1992) to match the Cardelli et al. (1989) model for $R_{\mathrm{V}}=2.51$ and $E_{\mathrm{B}-\mathrm{V}}=0.227$. In particular, we adopt $\lambda_{2175 \mathrm{~A}}=2170 \AA$ and $n_{\mathrm{FUV}}=5.5$, and we multiply the normalization parameters $a_{i}$ by additional factors $f_{i}: f_{\mathrm{FUV}}=1.5, f_{2175 \mathrm{~A}}=1.33$, and $f_{\mathrm{BKG}}=1.05$. In the second step, we turn off the "FUV" component of the Pei (1992) model for the $z=0.524$ galaxy by setting $f_{\mathrm{FUV}}=0$. This modification affects only the observed wavelengths shorter than $\sim 3300 \AA$, the location of the $2175 \AA$ feature redshifted by $z=0.524$, and is necessary to align the FUV spectra with the optical-NIR spectra (see Figure 7). We stress that extinction at longer wavelengths is very well constrained by the clear detection of a redshifted $2175 \AA$ feature by Junkkarinen et al. (2004), and thus cannot be increased. The resulting total extinction values $A_{\lambda}$ for the Swift/UVOT filters are-W2: 2.87; M2: 2.94; W1: 2.52; U: 2.71; B: 1.84; V: 1.46. For the remaining optical and near-IR filters, we use the values from Table 5 of Raiteri et al. (2005)—R: 1.26; I: 0.90; J: 0.46; $\mathrm{H}: 0.28 ; \mathrm{K}: 0.17$. We calculate the incident flux $F_{\text {inc }, \lambda}$ in the band corresponding to $\lambda$ from the observed (absorbed) flux $F_{\mathrm{abs}, \lambda}$ via $F_{\text {abs }, \lambda} / F_{\text {inc }, \lambda}=10^{A_{\lambda} / 2.5}$. The same corrections are applied to the ground-based optical data.

In addition, the source photometry is contaminated by the emission of a nearby AGN (named ELISA by Raiteri et al. 2005). Hence, we subtracted the ELISA contribution from the observed flux densities and then corrected for the combined extinction of both galaxies, following the prescriptions given by Raiteri et al. (2005) and Raiteri et al. (2008).

Likewise, the X-ray data need to be corrected for the effect of absorption. Here, the absorption effects of both our own Galaxy and the intervening $z=0.524$ system are considerable. Madejski et al. (1996) and Junkkarinen et al. (2004) argue that the absorption in the intervening system originates in material with abundances different from Galactic and, in reality, correct modeling of such absorption should take this into effect. However, as discussed by Madejski et al. (1996), the combined ROSAT and ASCA spectral fitting suggests that this effect is relatively modest, the joint $R O S A T-P S P C$ and $A S C A$ data are adequately fitted by an absorbing column of $(2.8 \pm 0.4) \times 10^{21} \mathrm{~cm}^{-2}$ located at $z=0$. Since the Swift $\mathrm{X}$-Ray Telescope (XRT) data have a somewhat lower signal-tonoise ratio $(\mathrm{S} / \mathrm{N})$ than the $A S C A$ observations, we simply adopt such a "local" model for absorption, since the main objective of our observations was to determine the underlying continuum of the AO $0235+164$ rather than the detailed spectral properties of the absorber. We note that this value is in fact consistent with the spectral fit to the Swift XRT data.

\subsection{GASP-WEBT}

The GLAST-AGILE Support Program (GASP) of the Whole Earth Blazar Telescope (WEBT) was initiated in 2007 with the aim of performing a long-term multi-wavelength monitoring of bright, $\gamma$-loud blazars (Villata et al. 2008b, 2009; D'Ammando et al. 2009; Raiteri et al. 2010). The GASP optical ( $R$ band), near-IR, and radio data are intended to complement the highenergy observations by the AGILE and Fermi (formerly GLAST) satellites.

AO $0235+164$ has been the target of several WEBT campaigns in the past (Raiteri et al. 2001, 2005, 2006, 2008) and it is now one of the GASP sources of highest observing priority. During the high $\gamma$-ray state observed in the second half of 2008, the source underwent an exceptional optical-to-radio outburst closely monitored by the GASP (Villata et al. 2008a, 2008c; Bach et al. 2008). The GASP optical data presented here were taken at the following observatories: Abastumani, Armenzano, Calar Alto, Crimean, Kitt Peak (MDM), L'Ampolla, Lulin, Roque de los Muchachos (KVA), San Pedro Martir, St. Petersburg, Talmassons, and Tuorla. Near-IR data in the $J$, $H$, and $K$ bands are all from Campo Imperatore. Millimeter $(\mathrm{mm})$ and centimeter $(\mathrm{cm})$ radio observations were performed at the SMA (230 and $345 \mathrm{GHz}$ ), Noto (43 GHz), Metsähovi (37 GHz), Medicina (5, 8, and $22 \mathrm{GHz}$ ), and UMRAO (4.8, 8.0, and $14.5 \mathrm{GHz}$ ) observatories. All IR, optical, and UV data are corrected for the effects of the intervening absorber (both due to the Milky Way and the intervening galaxy) as outlined above. 
Table 2

Observatories Participating in the Work, Periods of Observations, and Number of Data Points used in this Analysis

\begin{tabular}{|c|c|c|c|c|}
\hline Observatory & & Bands & Period of Observation & Data Points \\
\hline \multicolumn{5}{|c|}{ Radio } \\
\hline \multirow[t]{9}{*}{ GASP-WEBT } & Mauna Kea (SMA), USA & $345 \mathrm{GHz}$ & $54664-54840$ & 10 \\
\hline & & $230 \mathrm{GHz}$ & $54645-54842$ & 23 \\
\hline & Medicina, Italy & $5 \mathrm{GHz}$ & 54724 & 1 \\
\hline & & $8 \mathrm{GHz}$ & $54606-54777$ & 7 \\
\hline & & $22 \mathrm{GHz}$ & $54604-54779$ & 7 \\
\hline & Noto, Italy & $43 \mathrm{GHz}$ & $54642-54841$ & 8 \\
\hline & UMRAO, USA & $5 \mathrm{GHz}$ & $54677-54840$ & 16 \\
\hline & & $8 \mathrm{GHz}$ & $54621-54851$ & 20 \\
\hline & & $14.5 \mathrm{GHz}$ & $54633-54848$ & 41 \\
\hline \multirow[t]{10}{*}{ F-GAMMA } & Effelsberg 100-m & 2.64 & $54414-55227$ & 23 \\
\hline & & $4.85 \mathrm{GHz}$ & $54414-55227$ & 24 \\
\hline & & $8.35 \mathrm{GHz}$ & $54414-55227$ & 24 \\
\hline & & $14.6 \mathrm{GHz}$ & $54422-55227$ & 22 \\
\hline & & $23.05 \mathrm{GHz}$ & $54422-55227$ & 17 \\
\hline & & $32 \mathrm{GHz}$ & $54616-55227$ & 11 \\
\hline & & $42 \mathrm{GHz}$ & $54546-55227$ & 8 \\
\hline & IRAM 30-m & $86.2 \mathrm{GHz}$ & $54382-55228$ & 17 \\
\hline & & $142.3 \mathrm{GHz}$ & $54382-55228$ & 15 \\
\hline & & $228.4 \mathrm{GHz}$ & $54440-54806$ & 5 \\
\hline \multirow[t]{2}{*}{ IRAM PdBI } & & $88.9 \mathrm{GHz}$ & 54700 & 1 \\
\hline & & $169 \mathrm{GHz}$ & 54883 & 1 \\
\hline \multirow[t]{2}{*}{ OVRO } & & $15 \mathrm{GHz}$ & $54661-54848$ & 49 \\
\hline & & frared & & \\
\hline \multirow[t]{3}{*}{ GASP-WEBT } & Campo Imperatore & $J$ & $54645-54794$ & 87 \\
\hline & & $H$ & $54645-54794$ & 82 \\
\hline & & $K$ & $54645-54794$ & 83 \\
\hline Kanata & & $J$ & $54690-54753$ & 21 \\
\hline \multirow[t]{2}{*}{ SMARTS } & & $J$ & $54662-54847$ & 69 \\
\hline & & $K$ & $54662-54842$ & 39 \\
\hline & & ical & & \\
\hline \multirow[t]{12}{*}{ GASP-WEBT } & Abastumani $70 \mathrm{~cm}$ & $\mathrm{R}$ & $54687-54780$ & 287 \\
\hline & Armenzano, $40 \mathrm{~cm}$ & $R$ & $54699-54727$ & 16 \\
\hline & Calar Alto & $R$ & $54712-54887$ & 5 \\
\hline & Crimean 70cm; ST-7 & $R$ & $54691-54805$ & 218 \\
\hline & Kitt Peak (MDM $130 \mathrm{~cm}$ ) & $R$ & $54745-54801$ & 50 \\
\hline & L'Ampolla & $R$ & $54778-54784$ & 2 \\
\hline & Lulin (SLT) & $R$ & $54688-54862$ & 120 \\
\hline & Roque (KVA 35 cm) & $R$ & $54748-54862$ & 29 \\
\hline & San Pedro Martir $84 \mathrm{~cm}$ & $R$ & $54709-54773$ & 15 \\
\hline & St. Petersburg & $R$ & $54698-54865$ & 41 \\
\hline & Talmassons & $R$ & $54728-54843$ & 11 \\
\hline & Tuorla & $R$ & $54722-54732$ & 3 \\
\hline \multirow[t]{3}{*}{ SMARTS } & & $R$ & $54662-54868$ & 71 \\
\hline & & $B$ & $54662-54871$ & 69 \\
\hline & & $V$ & $54662-54859$ & 68 \\
\hline \multirow[t]{2}{*}{ Steward } & & $R$ & $54743-54832$ & 39 \\
\hline & & $V$ & $54743-54863$ & 44 \\
\hline \multirow[t]{3}{*}{ SWIFT-UVOT } & & $U$ & $54711-54818$ & 16 \\
\hline & & $B$ & $54711-54818$ & 16 \\
\hline & & $V$ & $54711-54818$ & 16 \\
\hline & & jiolet & & \\
\hline \multirow[t]{3}{*}{ SWIFT-UVOT } & & UVW1 & $54711-54818$ & 16 \\
\hline & & UVM2 & $54711-54818$ & 15 \\
\hline & & UVW2 & $54711-54818$ & 16 \\
\hline
\end{tabular}

\subsection{F-GAMMA}

During the 2008-2009 flaring period, quasi-simultaneous multi-frequency $\mathrm{cm} / \mathrm{mm}$ band (from $2.64 \mathrm{GHz}$ to $230 \mathrm{GHz}$ ) observations of AO 0235+164 were obtained using the Effelsberg $100 \mathrm{~m}$ and IRAM $30 \mathrm{~m}$ telescopes, within the framework of a Fermi related monitoring program of $\gamma$-ray blazars 
(F-GAMMA program; ${ }^{95}$ Fuhrmann et al. 2007; Angelakis et al. 2008).

The Effelsberg measurements were conducted with the secondary focus heterodyne receivers at 2.64, 4.85, 8.35, 10.45, 14.60, 23.05, 32.00, and $43.00 \mathrm{GHz}$. The observations were performed quasi-simultaneously with cross-scans, by slewing over the source position in the azimuth and elevation directions with an adaptive number of sub-scans chosen to reach the desired sensitivity (for details, see Fuhrmann et al. 2008; Angelakis et al. 2008). Consequently, pointing offset correction, gain correction, atmospheric opacity correction, and sensitivity correction have been applied to the data.

The IRAM 30 m observations were carried out with calibrated cross-scans using the single pixel heterodyne receivers B100, C150, B230 operating at 86.2, 142.3, and $228.4 \mathrm{GHz}$. The opacity-corrected intensities were converted into the standard temperature scale and finally corrected for small remaining pointing offsets and systematic gain-elevation effects. The conversion to the standard flux density scale was done using the instantaneous conversion factors derived from frequently observed primary (Mars, Uranus) and secondary $(\mathrm{W} 3(\mathrm{OH})$, K3-50A, NGC 7027) calibrators.

\subsection{OVRO}

Observations of $\mathrm{AO} 0235+164$ at $15 \mathrm{GHz}$ with the Owens Valley Radio Observatory (OVRO) $40 \mathrm{~m}$ telescope were made as part of an ongoing blazar monitoring program (Richards et al. 2011). The $40 \mathrm{~m}$ telescope is equipped with a cooled receiver at the prime focus, with a $3.0 \mathrm{GHz}$ bandwidth centered on 15.0 GHz and $2.5 \mathrm{GHz}$ noise-equivalent reception bandwidth. The receiver noise temperature is about $30 \mathrm{~K}$, and the total system noise temperature including $\mathrm{CMB}$, atmospheric, and ground contributions is about $55 \mathrm{~K}$. A dual off-axis corrugated horn feed projects to approximately Gaussian beams (157 arcsec full width at half-maximum, FWHM) on the sky, separated in azimuth by 12.95 arcmin. Dicke switching between the two beams is performed using the cold sky in the off-source beam as a reference, and a second level of switching is performed by alternating the source between the two beams to cancel atmospheric and ground noise. Calibration is achieved using a stable diode noise source for relative calibration and is referred to observations of 3C 286, for which we assume a flux density of $3.44 \mathrm{Jy}$ (Baars et al. 1977) with about 5\% absolute scale error. OVRO flux density measurements have a minimum uncertainty of $4 \mathrm{mJy}$ in $32 \mathrm{~s}$ of on-source integration, and a typical rms relative error of $3 \%$.

\subsection{IRAM Plateau de Bure Interferometer (PdBI)}

The Plateau de Bure Interferometer (PdBI; Winters \& Neri 2010 ) is able to observe in three atmospheric windows located around wavelengths of $1.3 \mathrm{~mm}, 2 \mathrm{~mm}$, and $3 \mathrm{~mm}$. Each of these bands covers a continuous range of frequencies that are available for observations; these ranges are $201-267 \mathrm{GHz}$ for the $1.3 \mathrm{~mm}$ band, $129-174 \mathrm{GHz}$ for the $2 \mathrm{~mm}$ band, and $80-116 \mathrm{GHz}$ for the $3 \mathrm{~mm}$ band.

Systematic monitoring of AGN is a by-product of regular observatory operations. The PdBI uses AGNs as phase and amplitude calibrators. Usually, one or two calibrators are measured every $\sim 20$ minutes for $\sim 2$ minutes (per source) throughout an observation. Antenna temperatures are converted into physical

\footnotetext{
95 http://www.mpifr-bonn.mpg.de/div/vlbi/fgamma/fgamma.html
}

flux densities using empirical antenna efficiencies as conversion factors. These factors are functions of frequencies and are located in the range from $\sim 22 \mathrm{Jy} \mathrm{K}^{-1}$ (for the $3 \mathrm{~mm}$ band) to $\sim 37 \mathrm{Jy} \mathrm{K}^{-1}$ (for the $1.3 \mathrm{~mm}$ band).

The PdBI is equipped with dual linear polarization Cassegrain focus receivers. This makes it possible to observe both orthogonal polarizations_-"horizontal" $(\mathrm{H})$ and "vertical" (V) with respect to the antenna frame-simultaneously. Due to the hardware layout of the correlators, it is not yet possible to observe all Stokes parameters. We collect linear polarization data on point sources via the Earth rotation polarimetry, i.e., we monitor the fluxes in the $\mathrm{H}$ and $\mathrm{V}$ channels as functions of parallactic angle $\psi$. The source polarization is derived from the parameterization

$$
q(\psi)=\frac{V-H}{V+H}(\psi) \equiv m_{L} \cos [2(\psi-\chi)] .
$$

Here, $m_{L}$ is the fraction of linear polarization (ranging from 0 to 1 ) and $\chi$ is the polarization angle (ranging from $0^{\circ}$ to $180^{\circ}$ and counted from north to east). For details, please refer to Trippe et al. (2010).

\subsection{Kanata}

We performed the $V-, J$-, and $K s$-band photometry and polarimetry of AO 0235+164 from 2008 August to 2008 October, using the TRISPEC instrument (Watanabe et al. 2005) installed at the $1.5 \mathrm{~m}$ Kanata telescope located at the Higashi-Hiroshima Observatory. TRISPEC has a CCD and two InSb arrays, enabling photo-polarimetric observations in one optical and two NIR bands simultaneously. We obtained 21 photometric data points in the $V, J, K s$ bands. A unit of the polarimetric observing sequence consisted of successive exposures at four position angles of the half-wave plates: $0^{\circ}, 45^{\circ}, 22.5,67.5$. The data were reduced according to the standard procedure of CCD photometry. We measured the magnitudes of objects with the aperture photometry technique. We performed differential photometry with a comparison star taken in the same frame of AO $0235+164$. Its position is R.A. $=02: 38: 32.31$, decl. $=+16: 35: 59.7$ (J2000) and its magnitudes are $V=12.720, J=11.248$, and $K s=$ 10.711 (Gonzalez-Perez et al. 2001; Cutri et al. 2003). The photometric data have been corrected for the Galactic extinction of $\mathrm{A}(V)=1.473, \mathrm{~A}(J)=0.458$, and $\mathrm{A}(K s)=0.171$, as explained in Section 3.1 .

We confirmed that the instrumental polarization was smaller than $0.1 \%$ in the $V$ band using observations of unpolarized standard stars, and hence we applied no correction for it. The zero point of the polarization angle is corrected as standard system (measured from north to east) by observing the polarized stars, HD19820 and HD25443 (Wolff et al. 1996).

\subsection{SMARTS}

AO $0235+164$ was observed at the Cerro Tololo InterAmerican Observatory (CTIO) as part of a photometric monitoring campaign of bright blazars with the Small and Moderate Aperture Research Telescope System (SMARTS). The source was observed with the SMARTS $1.3 \mathrm{~m}$ telescope and ANDICAM instrument (DePoy et al. 2003). ANDICAM is a dual-channel imager with a dichroic linked to an optical CCD and an IR imager, from which it is possible to obtain simultaneous data from 0.4 to $2.2 \mu \mathrm{m}$. Optical and near-infrared observations were taken in the $B, V, R, J$, and $K$ bands. 
Optical data were bias-subtracted, overscan-subtracted, and flat-fielded using the CCDPROC task in IRAF. Infrared data were sky-subtracted, flat-fielded, and dithered images were combined using in-house IRAF scripts. The raw photometry of comparison stars in the field of the blazar were calibrated using photometric zero points that were measured from 2008 to 2009 observations with ANDICAM of optical (Landolt 1992) and near-infrared (Persson et al. 1998) primary standards for each filter, correcting for atmospheric extinction derived from all the standards taken together. The averages of the comparison stars were used as a basis of differential photometry with respect to the blazar for all observations. Errors were determined by calculating the $1 \sigma$ variation in the magnitude of the comparison stars.

Fluxes were computed using values for Galactic extinction from Schlegel et al. (1998) and subtracting the nearby AGN "ELISA" as described in Raiteri et al. (2005). In addition, we accounted for the absorption of the $z=0.524$ system as outlined in Junkkarinen et al. (2004).

\subsection{Steward Observatory}

Optical spectropolarimetry and spectrophotometry of $\mathrm{AO}$ $0235+164$ during fall 2008 were provided by the monitoring program being conducted at Steward Observatory (Smith et al. 2009). This program utilizes the Steward Observatory CCD Spectropolarimeter (SPOL; Schmidt et al. 1992b) at either the $2.3 \mathrm{~m}$ Bok telescope located on Kitt Peak, AZ or the $1.54 \mathrm{~m}$ Kuiper telescope on Mt. Bigelow, AZ. The publicly available data ${ }^{96}$ include linear polarization and flux spectra (in first order) spanning 4000-7550 ̊. General data taking and reduction procedures used for this project are described in detail in Smith et al. (2003) and Smith et al. (2009). For the monitoring of AO $0235+164$, a $3^{\prime \prime}$ or $4^{\prime \prime}$ wide slit was used for spectropolarimetry, depending on the observing conditions, yielding a spectral resolution of $20-25 \AA$. An L-38 blocking filter was inserted into the collimated beam for all observations to prevent significant contamination from second-order light until well past $7600 \AA$. Total exposure times of between 24 and 80 minutes were used depending on the brightness of $\mathrm{AO} 0235+164$ and the sky/seeing conditions. Usually, a high signal-to-noise ratio measurement $(\mathrm{S} / \mathrm{N}>100)$ of the degree of polarization $(P)$ is determined from each observation by taking the median linear, normalized Stokes parameters $(q$ and $u$ ) in a $2000 \AA$ Åwide bin centered at $6000 \AA$. The reported values of $P$ have been corrected for statistical bias as in Wardle \& Kronberg (1974), but this correction is typically not significant because of the high $\mathrm{S} / \mathrm{N}$ of the binned data. The position angle (theta) of the optical linear polarization is calibrated by observing interstellar polarization standard stars (Schmidt et al. 1992a). Likewise, the flux spectra resulting from the spectropolarimetry are calibrated using observations of spectrophotometric standard stars (Massey et al. 1988). The flux spectra are corrected for atmospheric extinction using the standard extinction curves given in Baldwin \& Stone (1984) and Stone \& Baldwin (1983). Flux information for AO $0235+164$ was obtained through differential spectrophotometry of the blazar and a nearby field star ("Star 4"; Gonzalez-Perez et al. 2001). The spectrophotometry employed slits with widths of 7".6 or $12^{\prime \prime} .7$ to minimize seeingand color-dependent slit losses since the SPOL slit is left fixed in an east-west orientation on the sky and is not aligned with the parallactic angle. The wide-slit spectra of AO 0235+164 and

\footnotetext{
96 http://james.as.arizona.edu/ psmith/Fermi
}

Table 3

The log of Swift Observations Yielding Good XRT Data

\begin{tabular}{lclc}
\hline \hline $\begin{array}{l}\text { Date } \\
\text { (MJD-54000) }\end{array}$ & $\begin{array}{c}\text { Exposure } \\
(\mathrm{s})\end{array}$ & Photon Index & $\begin{array}{c}F_{2-10 \mathrm{keV}} \\
\left(10^{-12} \mathrm{erg} \mathrm{cm}^{-2} \mathrm{~s}^{-1}\right)\end{array}$ \\
\hline 711.4976 & 6876 & $1.91 \pm 0.09$ & $3.1 \pm 0.3$ \\
719.8695 & 1257 & 2 (assumed) & $3.2 \pm 0.4$ \\
737.9059 & 1448 & 2 (assumed) & $3.7 \pm 0.3$ \\
747.7476 & 2123 & 2 (assumed) & $4.3 \pm 0.3$ \\
758.7420 & 1133 & $2.44_{-0.08}^{+0.07}$ & $17.3 \pm 1.4$ \\
761.7541 & 1181 & $2.60 \pm 0.08$ & $15.0 \pm 1.2$ \\
781.0545 & 1144 & 2 (assumed) & $2.8 \pm 0.3$ \\
789.5603 & 1087 & 2 (assumed) & $4.1 \pm 0.4$ \\
803.7109 & 1175 & 2 (assumed) & $4.5 \pm 0.5$ \\
818.5249 & 1210 & 2 (assumed) & $4.8 \pm 0.4$ \\
\hline
\end{tabular}

Notes. In all cases, the spectrum was fitted with a power-law model absorbed by gas with Galactic abundances with a column of $2.8 \times 10^{21} \mathrm{~cm}^{-2}$ placed at $z=$ 0 : such an absorption form is only approximate, but it adequately fits $R O S A T$ and ASCA data, which in turn possess better signal to noise than individual Swift pointings (see the text). Since the quality of the data at MJD 54719, $54737,54747,54781,54789,54803$, and 54818 have too low an $\mathrm{S} / \mathrm{N}$ for reliable determination of spectrum, we assumed a photon index of two for those pointings.

the comparison star were convolved with standard filter transmission curves to determine differential magnitudes and derive the apparent magnitude of the blazar in the $V$ and $R$ bandpasses. The spectrophotometric observations were much shorter in duration (typically $<5$ minutes) than the spectropolarimetry, but of sufficient $\mathrm{S} / \mathrm{N}$ to be used to correct the much higher $\mathrm{S} / \mathrm{N}$ flux spectra of AO $0235+164$ resulting from the spectropolarimetry for any slit losses associated with the narrower slits used for those measurements.

\subsection{Swift XRT and UVOT}

AO 0235+164 was monitored as a result of an approved target of opportunity request by the Swift satellite (Gehrels et al. 2004), with weekly observations of $\approx 1-2 \mathrm{ks}$ performed from 2008 September 2 to 2008 December 18 (Table 3) with the XRT (Burrows et al. 2005) and with the Ultraviolet/Optical Telescope (UVOT; Roming et al. 2005).

The XRT data were reduced with the standard software (xrtpipeline v0.12.4) applying the default filtering and screening criteria (HEADAS package, v6.9 ${ }^{97}$ ). We extracted the XRT light curve in the $0.3-10 \mathrm{keV}$ energy band using the software tool xrtgrblc. The source events were extracted from circular regions centered on the source position. During the outburst we excluded the inner two pixels of the source to avoid pileup. Exposure maps were used to account for the effects of vignetting, PSF losses, and the presence of hot pixels and hot columns.

Since the source X-ray flux and spectrum are known to vary strongly, co-adding individual XRT observations could be misleading. We thus extracted the XRT data from each individual pointing separately, and fitted individual spectra using XSPEC. We rebinned the XRT data, requiring at least 25 counts in each new energy bin. As discussed in Section 3.1, we assumed that the combined Galactic and $z=0.524$ absorption is adequately described by a column of $2.8 \times 10^{21} \mathrm{~cm}^{-2}$ at $z=$ 0 : this is in fact consistent with the spectral fit to the Swift XRT data. We determined the unabsorbed X-ray flux by performing the spectral fit with fixed absorption, and then determining the

\footnotetext{
97 http://heasarc.gsfc.nasa.gov/lheasoft/
} 
Table 4

Results of Swift UVOT Observations of AO 0235+164

\begin{tabular}{|c|c|c|c|c|c|c|c|c|c|c|c|c|}
\hline Date & $\begin{array}{l}M \\
\text { (v) }\end{array}$ & $\begin{array}{c}F \\
(\mathrm{v})\end{array}$ & $\begin{array}{l}M \\
\text { (b) }\end{array}$ & $\begin{array}{c}F \\
\text { (b) }\end{array}$ & $\begin{array}{l}M \\
(\mathrm{u})\end{array}$ & $\begin{array}{c}F \\
(\mathrm{u})\end{array}$ & $\begin{array}{c}M \\
(\mathrm{w} 1)\end{array}$ & $\begin{array}{c}F \\
(\mathrm{w} 1)\end{array}$ & $\begin{array}{c}M \\
(\mathrm{~m} 2)\end{array}$ & $\begin{array}{c}F \\
(\mathrm{~m} 2)\end{array}$ & $\begin{array}{c}M \\
\text { (w2) }\end{array}$ & $\begin{array}{c}F \\
(\mathrm{w} 2)\end{array}$ \\
\hline 711.50 & $16.95 \pm 0.05$ & 2.32 & $17.93 \pm 0.04$ & 1.49 & $18.07 \pm 0.06$ & 1.04 & $18.16 \pm 0.06$ & 0.49 & $18.57 \pm 0.07$ & 0.43 & $18.93 \pm 0.06$ & 0.28 \\
\hline 719.87 & $16.79 \pm 0.09$ & 2.69 & $17.81 \pm 0.08$ & 1.65 & $17.75 \pm 0.11$ & 1.39 & $17.99 \pm 0.11$ & 0.57 & $18.23 \pm 0.16$ & 0.59 & $18.47 \pm 0.10$ & 0.42 \\
\hline 729.65 & $16.17 \pm 0.07$ & 4.76 & $17.03 \pm 0.06$ & 3.41 & $17.13 \pm 0.08$ & 2.46 & $17.18 \pm 0.08$ & 1.21 & $17.46 \pm 0.11$ & 1.20 & $17.85 \pm 0.08$ & 0.75 \\
\hline 737.91 & $16.29 \pm 0.06$ & 4.26 & $17.11 \pm 0.05$ & 3.17 & $17.11 \pm 0.07$ & 2.51 & $17.47 \pm 0.08$ & 0.93 & $17.62 \pm 0.11$ & 1.03 & $18.04 \pm 0.08$ & 0.63 \\
\hline 740.65 & $15.89 \pm 0.06$ & 6.18 & $16.80 \pm 0.05$ & 4.20 & $17.00 \pm 0.08$ & 2.77 & $17.17 \pm 0.08$ & 1.22 & $17.37 \pm 0.10$ & 1.30 & $17.78 \pm 0.08$ & 0.80 \\
\hline 747.75 & $16.17 \pm 0.05$ & 4.76 & $17.01 \pm 0.04$ & 3.46 & $17.14 \pm 0.06$ & 2.45 & $17.36 \pm 0.06$ & 1.03 & $17.43 \pm 0.07$ & 1.23 & $17.94 \pm 0.06$ & 0.69 \\
\hline 758.74 & $15.93 \pm 0.06$ & 5.95 & $16.86 \pm 0.05$ & 3.96 & $16.85 \pm 0.07$ & 3.18 & $16.83 \pm 0.07$ & 1.67 & $17.10 \pm 0.09$ & 1.66 & $17.61 \pm 0.07$ & 0.93 \\
\hline 761.75 & $16.05 \pm 0.06$ & 5.33 & $16.92 \pm 0.05$ & 3.76 & $16.99 \pm 0.07$ & 2.79 & $17.09 \pm 0.08$ & 1.31 & $17.29 \pm 0.09$ & 1.40 & $17.55 \pm 0.07$ & 0.99 \\
\hline 768.80 & $\ldots$ & $\ldots$ & $17.06 \pm 0.07$ & 3.30 & $17.08 \pm 0.10$ & 2.58 & $16.96 \pm 0.07$ & 1.47 & $\ldots$ & $\ldots$ & $17.45 \pm 0.12$ & 1.08 \\
\hline 780.31 & $17.07 \pm 0.16$ & 2.07 & $17.99 \pm 0.15$ & 1.40 & $17.91 \pm 0.19$ & 1.2 & $18.14 \pm 0.17$ & 0.50 & $18.17 \pm 0.28$ & 0.62 & $18.46 \pm 0.15$ & 0.43 \\
\hline 781.06 & $17.31 \pm 0.13$ & 1.66 & $17.85 \pm 0.09$ & 1.59 & $17.97 \pm 0.14$ & 1.13 & $18.08 \pm 0.13$ & 0.53 & $18.39 \pm 0.17$ & 0.51 & $18.63 \pm 0.11$ & 0.37 \\
\hline 789.56 & $16.63 \pm 0.08$ & 3.11 & $17.50 \pm 0.07$ & 2.21 & $17.46 \pm 0.10$ & 1.81 & $17.81 \pm 0.11$ & 0.68 & $17.98 \pm 0.16$ & 0.74 & $18.25 \pm 0.09$ & 0.52 \\
\hline 790.83 & $16.60 \pm 0.11$ & 3.20 & $17.40 \pm 0.09$ & 2.41 & $17.80 \pm 0.16$ & 1.32 & $17.73 \pm 0.14$ & 0.73 & $18.04 \pm 0.18$ & 0.70 & $18.34 \pm 0.13$ & 0.48 \\
\hline 803.71 & $17.66 \pm 0.16$ & 1.21 & $18.77 \pm 0.18$ & 0.69 & $18.63 \pm 0.21$ & 0.62 & $18.79 \pm 0.20$ & 0.27 & $18.51 \pm 0.17$ & 0.46 & $19.19 \pm 0.16$ & 0.22 \\
\hline 813.64 & $18.10 \pm 0.24$ & 0.81 & $18.87 \pm 0.21$ & 0.63 & $18.97 \pm 0.31$ & 0.45 & $18.96 \pm 0.24$ & 0.23 & $19.00 \pm 0.28$ & 0.29 & $19.00 \pm 0.16$ & 0.26 \\
\hline 818.52 & $17.43 \pm 0.15$ & 1.49 & $18.34 \pm 0.15$ & 1.01 & $18.87 \pm 0.32$ & 0.50 & $18.63 \pm 0.20$ & 0.32 & $19.07 \pm 0.23$ & 0.27 & $19.17 \pm 0.17$ & 0.22 \\
\hline
\end{tabular}

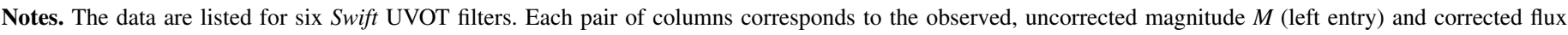

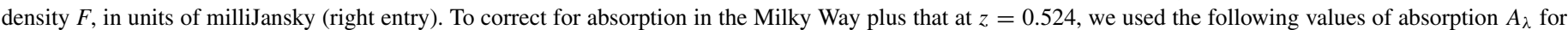

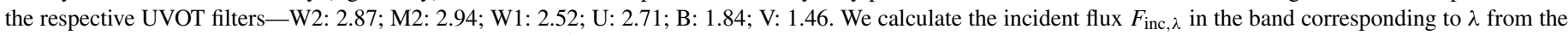
observed (absorbed) flux $F_{\mathrm{abs}, \lambda}$ via $F_{\mathrm{abs}, \lambda} / F_{\mathrm{inc}, \lambda}=10^{A_{\lambda} / 2.5}$. The same corrections are applied to ground-based optical data.

incident flux by forcing the absorption to be zero. We include those fluxes in the $2-10 \mathrm{keV}$ band in the last column of Table 3 . We note that the source was detected at a sufficiently good $\mathrm{S} / \mathrm{N}$ to determine the spectrum unambiguously only in the observations on MJD54711, MJD54758, and MJD54761. In other observations, we assumed a photon index of $\Gamma=2$, consistent with previous X-ray observations of this source in the low state, and note that the error resulting from such assumption on the inferred flux is comparable to the statistical error quoted in the last column of Table 3.

The UVOT photometry was done using the publicly available UVOT FTOOLS data reduction suite and is based on the UVOT photometric system described in Poole et al. (2008), but see also Breeveld et al. (2011) for an updated calibration. As discussed above, we adopted the corrections to the observed flux due to the absorption by the Milky Way plus the intervening galaxy at $z=0.524$ as outlined in Section 3.1. The results of Swift UVOT observations are presented in the Table 4.

\subsection{0. $R X T E$}

As part of our campaign, 30 observations of AO 0235+164 were obtained with Rossi X-ray Timing Explorer (RXTE) between 2008 October 18 and 2008 December 27. We analyzed the data from the Proportional Counter Array (PCA) following standard procedures. We selected only data from PCU2, the best-calibrated module and the only one which is always turned on. The data were screened in the following way: with a source elevation above the horizon $>10^{\circ}$, a pointing offset smaller than 0.02 , at least 30 minutes away from a South Atlantic Anomaly passage, and an electron contamination smaller than 0.1 . This resulted in a total exposure of $192.3 \mathrm{ks}$. Single net PCA exposures range from $2.1 \mathrm{ks}$ to $14.1 \mathrm{ks}$. Background was estimated with standard procedures and the detector response matrices extracted with the RXTE tools (command PCARSO v. 10.1).

For the spectral analysis, the fitting procedure was done with the XSPEC software package. The spectra from the channels corresponding to nominal energies of 2.6 to $10.5 \mathrm{keV}$ are adequately fitted by a single power-law model, absorbed by a fixed column of $2.8 \times 10^{21} \mathrm{~cm}^{-2}$ at $z=0$ as determined by the ROSAT and ASCA - in an analogous manner to the spectral fitting performed to the Swift XRT data above. The parameters of the fits are reported in Table 5; again, the last column reports the unabsorbed X-ray flux.

\section{VARIABILITY OF THE SOURCE}

\subsection{Multi-wavelength Light Curve}

In this section, we present the results of the multi-wavelength observations conducted on AO 0235+164 from 2008 August to 2009 January. Figure 3 shows the multi-wavelength data available. From the top to the bottom are: radio, near-infrared, optical, polarization degree (\%), polarization angle (deg), and $\mathrm{UV}, \mathrm{X}$-ray, and $\gamma$-ray from $100 \mathrm{MeV}$ to $100 \mathrm{GeV}$ data are grouped together.

The optical behavior is the best sampled among all. Two main flare peaks are visible in the period around 2008 October (MJD 54730-54750), and they are surrounded by other smaller peaks. The radio data show that the flux started to increase smoothly starting around the middle of 2007 (MJD 54500, which is apparent in Figure 4), reaching its maximum during the optical flare activity, and slowly decreasing when the source returned to a low-flux state in near-IR, optical, X-ray, and $\gamma$-ray bands. The near-infrared data show the same temporal trend as the optical bands. The UV data from Swift UVOT do not show the level of activity seen in the optical band. The X-ray data from Swift XRT and RXTE present a very pronounced peak clearly delayed with respect to the optical activity.

The Fermi light curve, as already discussed in Section 2.1, shows a broad high-state period followed by a final narrow peak succeeding the X-ray peak before getting to the low-flux state. Since then (up to the time of submitting this paper in autumn 2011), the source has been in a very quiet state.

Figure 4 shows the light curves constructed from the radio, $\mathrm{mm}$ and sub-mm data in an extended time interval, from 2007 to 2010 June (MJD 54400-55230). In the lowest energy band, the increasing trend of the flux started months before the 
Table 5

Best-fit Parameters for the PCA Data of each RXTE Observation with the Absorption Fixed at the Value Measured by ROSAT + ASCA, with the Column of $N_{\mathrm{H}}=28 \times 10^{20} \mathrm{~cm}^{-2}$ with Galactic Abundances

\begin{tabular}{|c|c|c|c|c|c|}
\hline Date & MJD-54000 & $\begin{array}{c}\text { Exposure } \\
\text { (s) }\end{array}$ & Photon Index $\Gamma$ & $\chi_{r}^{2} /$ dof & $F_{2-10}$ \\
\hline 2008 Oct 18 18:56 & 757.805 & 2688 & $2.46 \pm 0.13$ & $0.60 / 9$ & $2.00 \pm 0.08$ \\
\hline 2008 Oct 19 13:35 & 758.684 & 12416 & $2.55 \pm 0.06$ & $0.41 / 9$ & $2.09 \pm 0.04$ \\
\hline 2008 Oct 20 13:09 & 759.566 & 3024 & $2.73 \pm 0.14$ & $0.62 / 9$ & $1.79 \pm 0.07$ \\
\hline 2008 Oct $2114: 16$ & 760.647 & 6384 & $2.56 \pm 0.10$ & $0.38 / 9$ & $1.70 \pm 0.05$ \\
\hline 2008 Oct 22 13:53 & 761.597 & 3104 & $2.34 \pm 0.12$ & $0.56 / 9$ & $1.87 \pm 0.07$ \\
\hline 2008 Oct 23 18:08 & 762.773 & 2976 & $2.47 \pm 0.12$ & $0.58 / 9$ & $2.03 \pm 0.07$ \\
\hline 2008 Oct $2514: 08$ & 764.704 & 12656 & $2.57 \pm 0.07$ & $0.43 / 9$ & $1.77 \pm 0.04$ \\
\hline 2008 Oct 26 15:13 & 765.653 & 3136 & $2.71 \pm 0.14$ & $0.57 / 9$ & $1.68 \pm 0.08$ \\
\hline 2008 Oct 27 13:09 & 766.567 & 3200 & $2.35 \pm 0.16$ & $0.83 / 9$ & $1.41 \pm 0.07$ \\
\hline 2008 Oct 28 19:01 & 767.806 & 2320 & $2.71 \pm 0.21$ & $0.34 / 9$ & $1.26 \pm 0.08$ \\
\hline 2008 Oct 31 14:32 & 770.624 & 3152 & $2.24 \pm 0.27$ & $0.44 / 9$ & $0.71 \pm 0.06$ \\
\hline 2008 Nov 2 17:03 & 772.850 & 14448 & $2.26 \pm 0.18$ & $0.29 / 9$ & $0.53 \pm 0.03$ \\
\hline 2008 Nov 3 13:07 & 773.566 & 3200 & $2.46 \pm 0.40$ & $0.55 / 9$ & $0.54 \pm 0.07$ \\
\hline 2008 Nov 4 19:11 & 774.880 & 9072 & $2.72 \pm 0.36$ & $0.34 / 9$ & $0.37 \pm 0.04$ \\
\hline 2008 Nov 5 18:34 & 775.858 & 9680 & $2.12 \pm 0.31$ & $0.48 / 9$ & $0.35 \pm 0.04$ \\
\hline 2008 Nov 6 18:07 & 776.774 & 3200 & $1.91 \pm 0.55$ & $0.59 / 9$ & $0.32 \pm 0.06$ \\
\hline 2008 Nov 7 14:32 & 777.723 & 12992 & $2.50 \pm 0.38$ & $0.45 / 9$ & $0.28 \pm 0.03$ \\
\hline 2008 Nov 10 16:07 & 780.696 & 2880 & $3.53 \pm 0.79$ & $0.79 / 9$ & $0.38 \pm 0.09$ \\
\hline 2008 Nov 11 11:11 & 781.486 & 3264 & $2.37 \pm 0.61$ & $1.40 / 9$ & $0.27 \pm 0.06$ \\
\hline 2008 Nov 13 11:53 & 783.613 & 13168 & $2.37 \pm 0.38$ & $0.39 / 9$ & $0.25 \pm 0.03$ \\
\hline 2008 Nov 14 12:56 & 784.625 & 9888 & $2.39 \pm 0.36$ & $0.59 / 9$ & $0.30 \pm 0.04$ \\
\hline 2008 Nov 15 11:00 & 785.543 & 9904 & $2.37 \pm 0.36$ & $0.63 / 9$ & $0.31 \pm 0.04$ \\
\hline 2008 Nov 17 10:09 & 787.474 & 6336 & $2.50 \pm 0.59$ & $0.46 / 9$ & $0.24 \pm 0.05$ \\
\hline 2008 Nov 19 09:10 & 789.429 & 5568 & $2.94 \pm 0.44$ & $0.81 / 9$ & $0.39 \pm 0.05$ \\
\hline 2008 Nov 20 07:10 & 790.411 & 12192 & $1.99 \pm 0.36$ & $0.34 / 9$ & $0.26 \pm 0.03$ \\
\hline 2008 Nov 21 16:48 & 791.366 & 9744 & $2.92 \pm 0.63$ & $0.39 / 9$ & $0.23 \pm 0.04$ \\
\hline 2008 Nov 23 10:33 & 793.492 & 6432 & $2.08 \pm 0.68$ & $0.42 / 9$ & $0.21 \pm 0.04$ \\
\hline 2008 Dec 27 14:06 & 827.672 & 9664 & $2.69 \pm 0.50$ & $0.40 / 9$ & $0.25 \pm 0.04$ \\
\hline
\end{tabular}

Notes. Description of columns: (1) and (2) Observing date, (3) Exposure (s), (4) photon index and error, (5) reduced $\chi^{2}$ and No. of degrees of freedom, and (6) Flux in the $2-10 \mathrm{keV}$ band, in units of $10^{-11} \mathrm{erg} \mathrm{cm}^{-2} \mathrm{~s}^{-1}$.

increased level of activity seen in the optical and higher energy bands. After the period of the increased radio/mm flux associated with the optical flaring activity, the source enters a period of gradually declining flux.

\subsection{Cross-correlation Studies and Time Delays}

We searched for correlations of variability between different bands, with the goal to understand the relationship between the fluxes of AO $0235+164$ at different energies. The crosscorrelation studies between the optical $R$ band and $\gamma$-ray fluxes are illustrated in the top panel of Figure 5. Those data have a Spearman correlation coefficient ${ }^{98}$ of 0.75 (Spearman 1904). The relations between the $\gamma$-ray and $230 \mathrm{GHz}$ and $345 \mathrm{GHz}$ fluxes have also been evaluated and the results are shown in Figure 5 in the bottom panel: the Spearman correlation coefficient between $\gamma$-ray fluxes and $230 \mathrm{GHz}$ data is 0.70 , showing that there exists a correlation between the two data sets. On the other hand, the sampling at $345 \mathrm{GHz}$ is poor, with only a few data points at that frequency and the evaluation of a correlation has not been performed. In all cases, no correlation is found at $90 \%$ confidence level. We note here that Agudo et al. (2011b), using data collected for this object over a longer time span than that covered by our observations, performed a lightcurve correlation analysis following the method described by Agudo et al. (2011a). They found that these bands are correlated

\footnotetext{
98 Wessa, P. (2011), Free Statistics Software, Office for Research Development and Education, version 1.1.23-r7, http://www.wessa.net/
}

at a $99.7 \%$ confidence level. In our case, no correlation is found at $90 \%$ confidence level: it is very likely that the stronger correlation of signals derived by Agudo et al. (2011b) is caused by their use of a significantly longer time span, amounting to roughly eight years. All this suggests that the variability of the source in the $\gamma$-ray and radio-to-mm regimes on long timescales is correlated, but the situation on shorter timescales is less clear.

Since the time series in the optical $R$ band and $\gamma$-ray are the best sampled in this study, it was possible to calculate lags/leads between those bands. To this end, we calculated the discrete correlation function (DCF; Edelson \& Krolik 1988). We binned the data sets in order to smooth the intraday features in the optical light curves and to obtain similar sampling in the $\gamma$-ray band. We tried several bin sizes from one to seven days to check how sensitive the results are to this smoothing procedure. The DCF from the optical and $\gamma$-ray data do not show significant peaks on short timescales (one day) meaning no optical $-\gamma$ correlation is detected over the observing period. Figure 6 shows the result of this DCF analysis when the light curves are binned over one day. A peak can be seen at 15 days (with optical lagging $\gamma$-rays), however the significance is modest. Agudo et al. (2011b) found that, for a similar period, the optical flux lags the $\gamma$-rays by $\sim 10$ days, but their DCF peak is much broader, and could be interpreted as being consistent with no lag.

\subsection{Time Dependence of Optical Polarization}

As illustrated in Figure 3, the polarization degree and angle are highly variable; the former correlates with the optical flux 


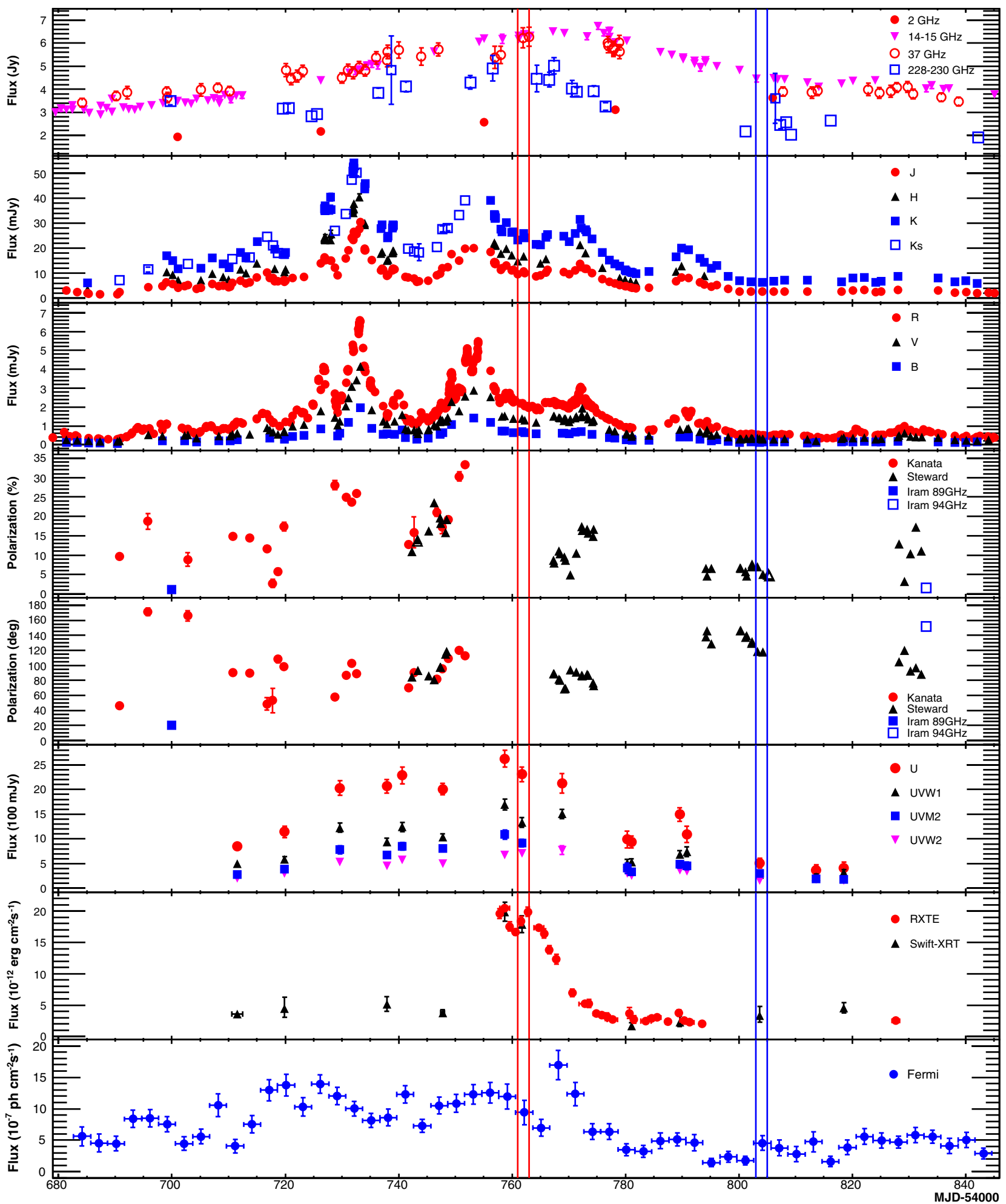

Figure 3. AO 0235+164 light curve from 2008 August 4 to 2009 February 4 in different energy ranges. From the top to the bottom: radio, near IR, optical, UV, X-rays, and $\gamma$-rays above $100 \mathrm{MeV}$. Panels 4 and 5 from the top report the polarization data from the Kanata optical observatory and IRAM radio telescope. Two double vertical lines mark the epochs for which we extracted the SEDs modeled in Section 6.

(A color version of this figure is available in the online journal.)

and at the two largest flux peaks reaches values of $25 \%$ and $35 \%$, respectively. This correlation was studied over a longer period of time (from 2008 August 12 to 2009 February 18) by Sasada et al. (2011), and in the past, during the outburst of 2006 December, by Hagen-Thorn et al. (2008). A trend of the stabilization of the polarization angle during flares is seen both in 2006 and 2008, but around different values, with the electric vector polarization angle (EVPA) at $\sim-30^{\circ}$ and $\sim 100^{\circ}$, respectively. Comparing EVPA with the position angle of the parsec-scale jets, HagenThorn et al. (2008) found that there is a trend of their alignment during high states. However, since the parsec-scale jet in $\mathrm{AO}$ $0235+164$ shows large changes of direction with time (Jones 

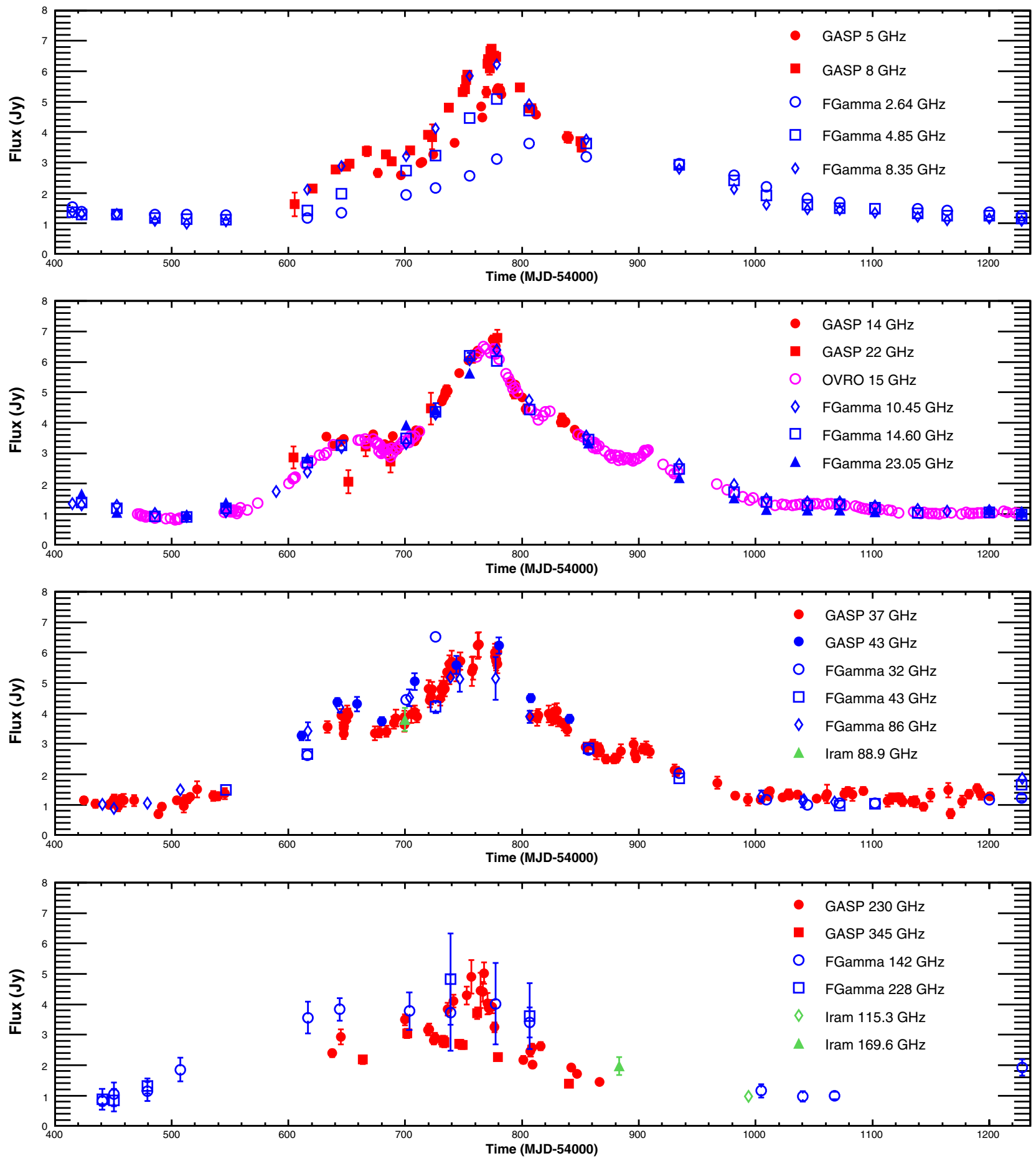

Figure 4. Radio, millimeter, and submillimeter light curves of AO 0235+164 from mid 2007 until 2010 June (MJD 54400-55230). In these energy bands, the flux began to increase around the middle of 2007 (MJD 54500), months before the start of the optical and higher energy activity of the source.

(A color version of this figure is available in the online journal.)

et al. 1984; Chu et al. 1996; Jorstad et al. 2001; Piner et al. 2006) and the jet direction to which EVPA was compared was inferred from the VLBI maps taken in different epochs, the claimed alignment could be accidental. Indeed, comparison of EVPA during flux peaks in 2008 with the direction of the jet determined during the same epoch by VLBI observations does not confirm such an alignment (Agudo et al. 2011b). On the contrary, both angles are oriented perpendicular rather than parallel to each other, albeit with a large scatter, with EVPA at optical flux peaks $\sim 100^{\circ}$ versus $\chi_{\text {jet }} \sim 0^{\circ}$. This implies a parallel orientation of the magnetic fields to the jet and may indicate production of flares in a reconfinement shock (Nalewajko 2009).

\section{BROADBAND SPECTRAL ENERGY DISTRIBUTION}

Our unprecedented time sampling of AO 0235+164 in several spectral bands allows us to extract accurate instantaneous SEDs, which are needed to correctly interpret the broadband emission of the source. We reiterate that in order to build the intrinsic SED and correctly convert the observed magnitudes to de-absorbed fluxes, extinction must be taken into account, including both 

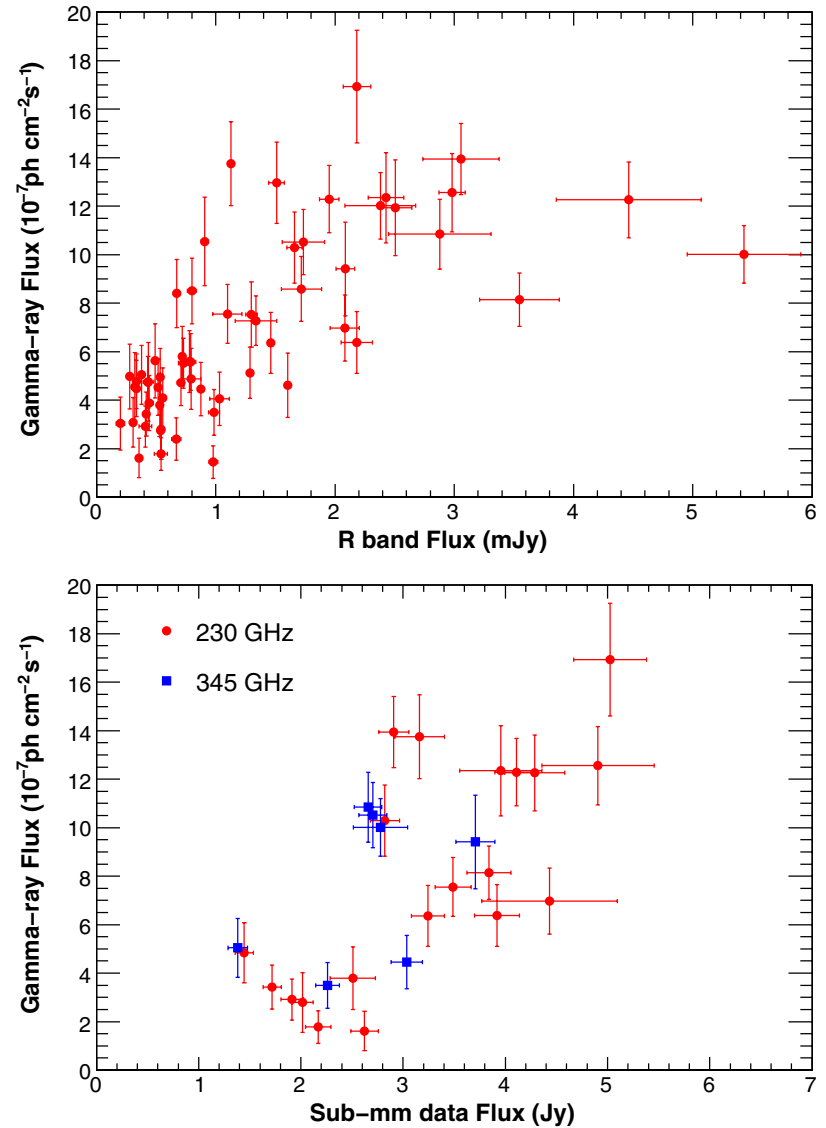

Figure 5. Top: plot of the $\gamma$-ray flux vs. $R$-band flux; both fluxes are averaged in three day time intervals. The data suggest that $\gamma$-ray and optical fluxes follow each other, but the correlation is small with $\gamma$-ray flux reaching a plateau at the level $\sim 1.2 \times 10^{-6}$ when the optical flux reaches $\sim 3 \mathrm{mJy}$, but not increasing beyond $\sim 1.2 \times 10^{-6}$ when the optical flux increases to $\sim 5 \mathrm{mJy}$. Bottom: similar plot of $\gamma$-ray flux vs. high-frequency radio-band flux; both fluxes are averaged in three day time intervals. Likewise, there is a general trend of increase in both bands, but the correlation is small.

(A color version of this figure is available in the online journal.)

Galactic extinction, and that due to the $z=0.524$ system. We discussed in Section 3.1 how the IR, optical, UV data, and soft X-rays are affected by the absorption due to both systems. Following the prescriptions from Junkkarinen et al. (2004), we assume that the soft $\mathrm{X}$-ray absorption is adequately described by a column density of $2.8 \times 10^{21} \mathrm{~cm}^{-2}$ at $z=0$. However, for the far-UV data, we use a modified extinction model based on the work of Pei (1992).

Figure 7 shows the broadband SEDs obtained by plotting simultaneous radio, NIR, optical, UV, X-ray data in the following two 2 day epochs.

1. MJD 54761-54763 (shown in red) corresponding to the maximum of the X-ray flare, coincident with a highly variable near-IR/optical/UV state and a high $\gamma$-ray state.

2. MJD 54803-54805 (shown in blue) corresponding to a low state in all bands, following the high-activity period.

The plotted data points were extracted from the larger data sets as follows.

1. Radio data. Most of the data points are simultaneous measurements. Although the sampling at some particular wavelengths is poor, the available radio light curves show very smooth and slow trends, thus we have also plotted interpolated values based on the extended data set of about 10 days in length, centered on the main observation period.

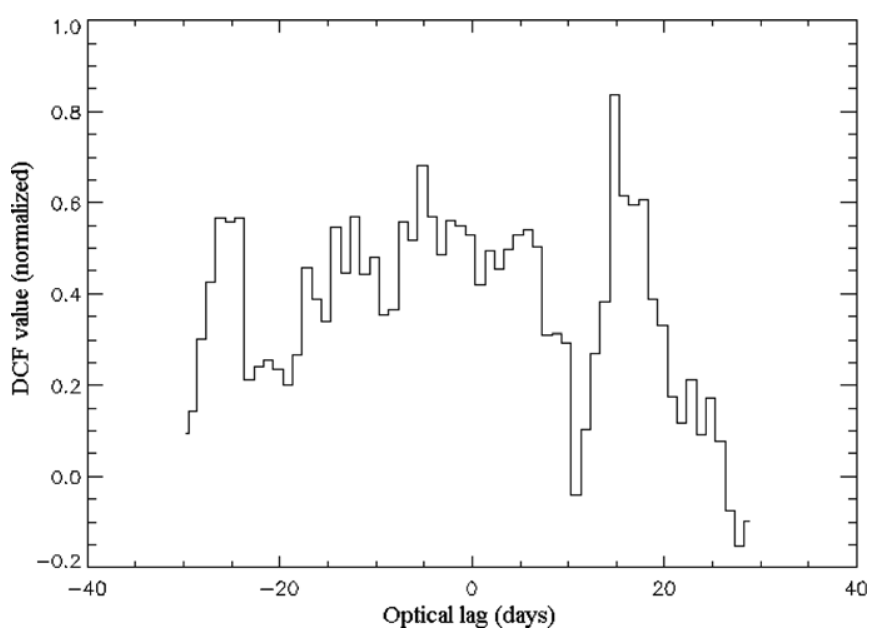

Figure 6. Discrete correlation function (DCF) calculated between optical $R$ band and Fermi-LAT $\gamma$-ray data binned over one day intervals. Positive values correspond to $\gamma$-rays leading the optical signal.

2. Near-IR/optical/UV. All the data shown are simultaneous measurements made by GASP-WEBT, SMARTS, and Swift UVOT telescopes.

3. X-ray data. In the first epoch (MJD 54761-54763), we present the Swift XRT observation with a butterfly plot. In the second epoch (MJD 54803-54805), the S/N for the Swift XRT measurement is too low to allow a good spectral representation and only the flux upper limit is reported.

4. $\gamma$-ray data. The $\gamma$-ray spectra have been built following the analysis procedure described in Section 2.2. Since the time intervals chosen to build the broadband SED are too short to allow a good reconstruction of the $\gamma$-ray spectrum, longer periods have been used. The first time interval, MJD 54750-54770, corresponds to a period of high $\gamma$-ray emission, which includes the X-ray flare period. The high state is followed by a lower emission state whose spectrum is averaged in the interval MJD 54780-54840.

The overall SED, plotted in Figure 7, appears quite similar to that measured for other blazars. There is one marked difference: at least for the first period, the X-ray spectrum is soft, yet it is not located on the extrapolation of the optical/UV spectrum. For this to be the case, extinction would have to be significantly greater, with the error at the level of at least $50 \%$, which we consider unlikely. Assuming that we adopted the correct extinction, the broadband SED does show a distinct feature in the soft X-ray band, separate from the two broad peaks forming the SED in most blazars, and we discuss its origin below.

\section{MODELING OF THE BROADBAND SPECTRUM}

AO 0235+164, like many other luminous, low-frequencypeaked BL Lac objects, shows broad emission lines (BELs; Cohen et al. 1987; Nilsson et al. 1996; Raiteri et al. 2007). Using the emission-line spectrum reported in Raiteri et al. (2007), correcting the line flux for extinction, and assuming that the contribution of the lines measured by them to the total luminosity of BELs is the same as in the composite spectrum of quasars (Francis et al. 1991), we find $L_{\mathrm{BEL}} \sim 4 \times 10^{44} \mathrm{erg} \mathrm{s}^{-1}$. For the typical covering factor of the broad-line region (BLR) $\xi_{\mathrm{BEL}} \sim 0.1$, this implies a luminosity of the accretion disk of $L_{d} \sim 4 \times 10^{45} \mathrm{erg} \mathrm{s}^{-1}$. With such a high accretion luminosity, if observed directly, i.e., without being overshone by the jet nonthermal radiation, AO $0235+164$ would satisfy a formal 


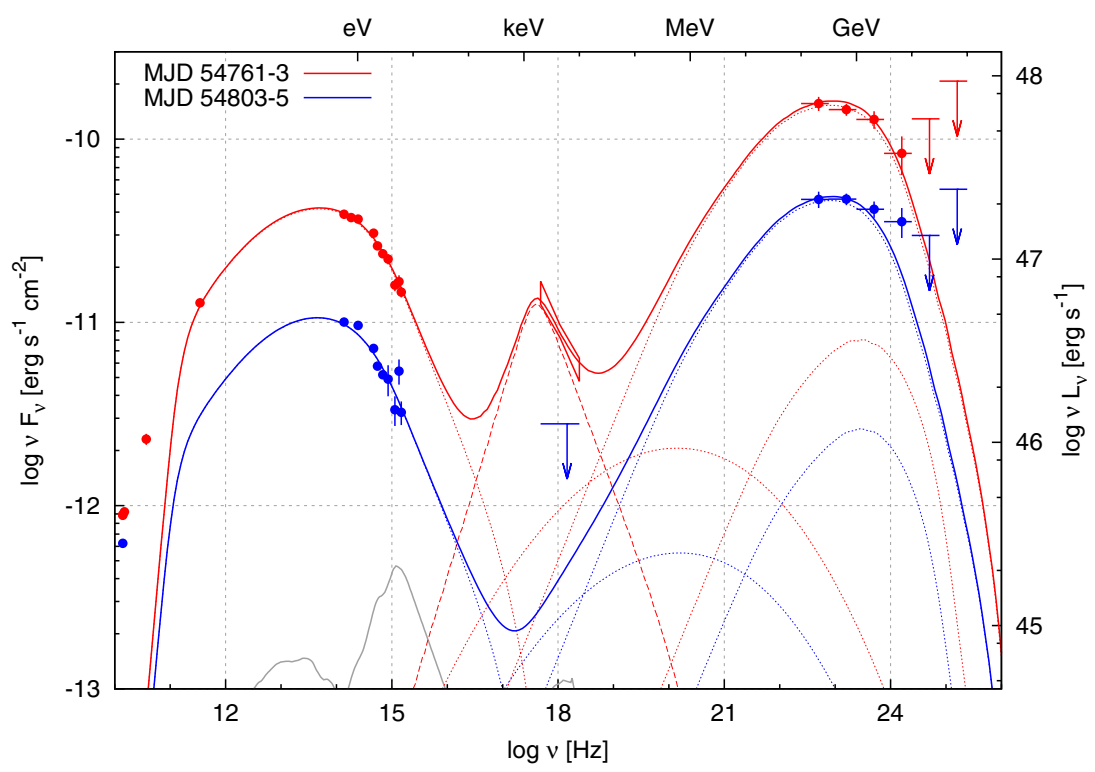

Figure 7. Numerical models fitted to observed spectral states of AO $0235+164$, dominated energetically by the Comptonization of the infrared radiation from the dusty torus (ERCIR). Red lines show a fit to the high state (MJD 54761-3), including the bulk-Compton feature (dashed line). Blue lines show a fit to the low state (MJD 54803-5). Dotted lines indicate individual spectral components, in order of increasing peak frequencies: synchrotron, SSC, ERCIR, ERCBEL. Solid lines show the sums of all individual components. Note that presented models do not cover the radio production which at $v<100 \mathrm{GHz}$ is strongly synchrotron-self-absorbed for our source parameters and must originate at much larger distances from the BH than a few parsecs. The gray line shows the quasar composite SED adopted from Elvis et al. (1994) and normalized to the accretion disk luminosity $L_{d}=4 \times 10^{45} \mathrm{erg} \mathrm{s}^{-1}$.

(A color version of this figure is available in the online journal.)

criterion to be classified as a quasar (see also Murphy et al. 1993). This means that, according to the AGN unification models, it should possess a typical dusty torus, a strong source of thermal infrared radiation (IR) with a typical covering factor of $\xi_{\text {IR }} \sim 0.1$ (e.g., Haas et al. 2004). Recent mid-IR interferometric observations for a sample of nearby AGNs show that such tori can extend beyond $10 \mathrm{pc}$ from the central BH (Tristram \& Schartmann 2011). The mass of the $\mathrm{BH}$ in this object is likely to be in the range $M_{\mathrm{BH}} \sim 2-6 \times 10^{8} M_{\odot}$ (Liu et al. 2006; Raiteri et al. 2007; Wu et al. 2011), which implies the Eddington ratio $L_{d} / L_{\mathrm{EDD}} \geqslant 0.1$.

In order to determine which process dominates the highenergy emission, whether it is ERC or SSC, one can estimate their luminosity ratio as $L_{\mathrm{ERC}} / L_{\mathrm{SSC}} \simeq u_{\mathrm{ext}}^{\prime} / u_{\text {syn }}^{\prime}$, where $u_{\text {ext }}^{\prime}$ is the comoving energy density of the external radiation, which, depending on the source location, could be dominated either by $u_{\mathrm{BEL}}^{\prime}$ or $u_{\mathrm{IR}}^{\prime}$, and $u_{\mathrm{syn}}^{\prime}$ is the comoving energy density of the synchrotron radiation. These energy densities scale like $u_{\mathrm{BEL}(\mathrm{IR})}^{\prime} \simeq \Gamma_{\mathrm{j}}^{2} u_{\mathrm{BEL}(\mathrm{IR})} \simeq \Gamma_{\mathrm{j}}^{2} \xi_{\mathrm{BEL}(\mathrm{IR})} L_{\mathrm{d}} /\left(4 \pi r_{\mathrm{BEL}(\mathrm{IR})}^{2} c\right)$ for $r \leqslant r_{\mathrm{BEL}(\mathrm{IR})}$, respectively, and $u_{\mathrm{syn}}^{\prime} \simeq L_{\mathrm{syn}} /\left(4 \pi R^{2} \mathcal{D}^{4} c\right)$, where $r_{\mathrm{BEL}} \sim 0.1\left(L_{\mathrm{d}, 46}\right)^{1 / 2} \mathrm{pc}$ is the characteristic radius of the BLR, $r_{\mathrm{IR}} \sim 2.5\left(L_{\mathrm{d}, 46}\right)^{1 / 2} \mathrm{pc}$ is the inner radius of the dusty torus, $R$ is the emitting zone radius related to its distance by $r=R \Gamma_{\mathrm{j}}, \Gamma_{\mathrm{j}}=$ $\left(1-\beta_{\mathrm{j}}^{2}\right)^{-1 / 2}$ is the jet Lorentz factor, and $\beta_{\mathrm{j}}$ is the jet velocity in units of $c$ (Sikora et al. 2009). Considering the emitting zone located at either characteristic radius, i.e., $r \simeq r_{\mathrm{BEL}(\mathrm{IR})}$, and neglecting the distinction between the Doppler factor $\mathcal{D}$ and the Lorentz factor $\Gamma_{\mathrm{j}}$, we obtain $L_{\mathrm{ERC}} / L_{\mathrm{SSC}} \simeq \xi_{\mathrm{BEL}(\mathrm{IR})} \Gamma_{\mathrm{j}}^{4}\left(L_{\mathrm{d}} / L_{\mathrm{syn}}\right)$. In the case of AO $0235+164$, we observe $L_{\mathrm{d}} / L_{\text {syn }} \sim 0.01$ and thus $L_{\mathrm{ERC}} / L_{\mathrm{SSC}} \simeq 160\left(\xi_{\mathrm{BEL}(\mathrm{IR})} / 0.1\right)\left(\Gamma_{\mathrm{j}} / 20\right)^{4}$. Hence, even for a moderate bulk Lorentz factor, in order for the SSC component to dominate the ERC component, one requires covering factors two orders of magnitude lower than typically assumed in quasars.
In this section, we verify the ERC scenario by fitting the observed SEDs with one-zone leptonic models (Moderski et al. 2003). We follow the evolution of relativistic electrons injected into a thin spherical shell propagating conically with a constant Lorentz factor $\Gamma_{\mathrm{j}}$ undergoing adiabatic and radiative losses due to the synchrotron and inverse Compton emission. The external radiation includes BELs of characteristic photon energy $E_{\mathrm{BEL}} \sim 10 \mathrm{eV}$ and infrared dust radiation of characteristic energy $E_{\mathrm{IR}} \sim 0.3 \mathrm{eV}$. We attempted to fit the high state of MJD 54761-3 with a "blazar zone" located either within (ERCBEL model) or outside the BLR (ERCIR model). In the ERCBEL model, the electron break inferred from the synchrotron spectrum is too low to reproduce the $\gamma$-ray spectrum above $\sim 1 \mathrm{GeV}$. This problem is absent in the ERCIR model (red lines in Figure 7). This is because Comptonization of IR photons is subject to much weaker Klein-Nishina suppression in the $\mathrm{GeV}$ band than Comptonization of optical/UV emissionline photons. The parameters of the ERCIR model are: location $r=r_{\mathrm{IR}}$, Lorentz factor $\Gamma_{\mathrm{j}}=20$, opening angle $\theta_{\mathrm{j}}=1 / \Gamma_{\mathrm{j}}=2.9$ (hence the Doppler factor $\mathcal{D}_{\mathrm{j}}=\Gamma_{\mathrm{j}}$ ), magnetic field strength $B^{\prime}=0.22 \mathrm{G}$, and viewing angle $\theta_{\mathrm{obs}}=2.3$. Electrons are injected with a doubly broken energy distribution with $\gamma_{\mathrm{br}, 1}=100, \gamma_{\mathrm{br}, 2}=5800, p_{1}=1.5, p_{2}=2.03$, and $p_{3}=3.9$.

The rate of electron energy injection is $\dot{E}_{\mathrm{e}, \mathrm{inj}}^{\prime} \sim 4.8 \times$ $10^{43} \mathrm{erg} \mathrm{s}^{-1}$. Over comoving time $\Delta t^{\prime} \sim r_{\mathrm{IR}} /\left(2 \Gamma_{\mathrm{j}} \beta_{\mathrm{j}} c\right) \sim$ $4 \times 10^{6} \mathrm{~s}$, the total injected electron energy is $E_{\mathrm{e}, \text { inj }}^{\prime} \sim \dot{E}_{\mathrm{e}, \text { inj }}^{\prime} \Delta t^{\prime} \sim$ $1.9 \times 10^{50} \mathrm{erg}$. At the end of the injection, the total number of electrons is $N_{\mathrm{e}}=6.6 \times 10^{54}$ and their total energy in the comoving frame is $E_{\mathrm{e}}^{\prime} \sim 1.1 \times 10^{50} \mathrm{erg}$. The average efficiency of electron energy losses is $\eta_{\mathrm{e}, \text { loss }}=1-\left(E_{\mathrm{e}}^{\prime} / E_{\mathrm{e}, \text { inj }}^{\prime}\right) \sim 0.42$. The electron flux is $\dot{N}_{\mathrm{e}} \sim \pi \Gamma_{\mathrm{j}} R^{2} c N_{\mathrm{e}} / V^{\prime} \sim 1.2 \times 10^{49} \mathrm{~s}^{-1}$, where $V^{\prime} \sim 4 \pi R^{3} / 3$ is the volume of the emitting region in the comoving frame and $R \sim \theta_{\mathrm{j}} r$ is the jet radius. The electron energy flux is $L_{\mathrm{e}} \sim \pi \Gamma_{\mathrm{j}}^{2} R^{2} c E_{\mathrm{e}}^{\prime} / V^{\prime} \sim 4.1 \times 10^{45} \mathrm{erg} \mathrm{s}^{-1}$ 
and the proton energy flux is $L_{\mathrm{p}} \sim \pi \Gamma_{\mathrm{j}}^{2} R^{2} c N_{\mathrm{p}} m_{p} c^{2} / V^{\prime} \sim$ $3.6 \times 10^{47}\left(n_{p} / n_{e}\right)$ erg s$^{-1}$, where $N_{\mathrm{p}} \sim N_{\mathrm{e}}\left(n_{p} / n_{e}\right)$ is the total number of protons and $\left(n_{e} / n_{p}\right)$ is the lepton-to-proton number ratio. The magnetic energy flux is $L_{\mathrm{B}}=\pi \Gamma_{\mathrm{j}}^{2} R^{2} c u_{\mathrm{B}}^{\prime} \sim$ $4.4 \times 10^{45} \mathrm{erg} \mathrm{s}^{-1}$. The resulting jet magnetization parameter is $\sigma_{\mathrm{B}} \sim L_{\mathrm{B}} / L_{\mathrm{p}} \sim 0.012\left(n_{e} / n_{p}\right)$, and the radiative efficiency is $\eta_{\mathrm{rad}, \mathrm{j}} \sim L_{\mathrm{obs}} /\left(2 \Gamma_{\mathrm{j}}^{2} L_{\mathrm{p}}\right) \sim 0.022\left(n_{e} / n_{p}\right)$, where $L_{\mathrm{obs}} \sim$ $6.4 \times 10^{48} \mathrm{erg} \mathrm{s}^{-1}$ is the observed bolometric luminosity of the source. The relation between the jet power and accretion disk luminosity is $L_{\mathrm{p}} / L_{\mathrm{d}} \sim 91\left(n_{p} / n_{e}\right)$. Parameterizing the jet production efficiency by $L_{\mathrm{p}} \sim \eta_{\mathrm{j}} \dot{M}_{\mathrm{acc}} c^{2}$ and the radiative efficiency of the accretion disk $L_{\mathrm{d}} \sim \eta_{\mathrm{rad}, \mathrm{d}} \dot{M}_{\text {acc }} c^{2}$, where $\dot{M}_{\text {acc }}$ is the accretion rate, we obtain $n_{e} / n_{p} \sim 91\left(\eta_{\mathrm{rad}, \mathrm{d}} / \eta_{\mathrm{j}}\right)$. For $\eta_{\text {rad,d }} \sim 0.1$ and $\eta_{\mathrm{j}} \sim 1$, we have $n_{e} / n_{p} \sim 9.1$ and hence $\sigma_{\mathrm{B}} \sim 0.11$ and $\eta_{\mathrm{rad}, \mathrm{j}} \sim 0.2$. We are thus able to match the jet power with the accretion power onto the central $\mathrm{BH}$, adopting a reasonably low jet magnetization, which allows formation of strong shock waves. The ratio of electrons to protons is consistent with the results of Sikora \& Madejski (2000). This model predicts the observed variability timescale of $t_{v} \simeq(1+z) R /\left(c \Gamma_{\mathrm{j}}\right) \sim 8$ days, consistent with the timescale of the significant variations of the optical and $\gamma$-ray flux.

As we noted above, the X-ray spectrum, at least during the first of the two epochs considered here, is too soft to be interpreted as an SSC component and cannot be the highenergy tail of the synchrotron component since it does not lie on the extrapolation of the optical-UV spectrum (but see the caveats above, related to the corrections for extinction). Instead, it can be explained by Comptonization of external radiation by a population of relatively cold electrons (Begelman \& Sikora 1987; Ravasio et al. 2003). Such bulk Compton radiation is expected to be produced in a jet much closer to the $\mathrm{BH}$ than the nonthermal blazar radiation, at distances at which cooling of even mildly relativistic electrons is very efficient. There, the jet is still in the acceleration phase and, therefore, its bulk Lorentz factor is expected to be smaller than in the blazar zone. But noting that according to magnetohydrodynamical models the acceleration process is very smooth (see, e.g., Komissarov et al. 2007) and that the bulk Compton radiation must be significantly Doppler boosted to be visible in the blazar spectra, the dominant contribution to bulk-Compton radiation is expected to be produced at distances which are already well separated from the base of the jet (Sikora et al. 2005; Celotti et al. 2007). We consider a stationary emitting region at a characteristic radius of $r_{\mathrm{b}} \sim 100 R_{\mathrm{g}} \sim 6 \times 10^{15} \mathrm{~cm}$, where $R_{\mathrm{g}}=G M_{\mathrm{BH}} / c^{2}$ is the gravitational radius of the central $\mathrm{BH}$ of mass $M_{\mathrm{BH}} \sim 4 \times 10^{8} M_{\odot}$. The bulk Lorentz factor is $\Gamma_{\mathrm{b}} \sim 10$ and the Doppler factor is $\mathcal{D}_{\mathrm{b}}=1 /\left[\Gamma_{\mathrm{b}}\left(1-\beta_{\mathrm{b}} \cos \theta_{\mathrm{obs}}\right)\right] \sim 16$. Bulk-Compton luminosity is given by the approximate formula

$$
L_{\mathrm{b}} \simeq N_{\mathrm{e}, \mathrm{b}}\left|\dot{E}_{\mathrm{e}, \mathrm{IC}}\right|_{\mathrm{b}} \frac{\mathcal{D}_{\mathrm{b}}^{3}}{\Gamma_{b}},
$$

where $N_{\mathrm{e}, \mathrm{b}}$ is the number of electrons enclosed in the $\Delta r \sim r_{\mathrm{b}}$ portion of a jet, $\left|\dot{E}_{\mathrm{e}, \mathrm{IC}}\right|_{\mathrm{b}}=(4 / 3) c \sigma_{T} u_{\mathrm{ext}, \mathrm{b}} \Gamma_{\mathrm{b}}^{2}$ is the rate of production of Compton radiation by a single electron, and $u_{\mathrm{ext}, \mathrm{b}}=\xi_{\mathrm{b}} L_{\mathrm{d}} /\left(4 \pi r_{\mathrm{b}}^{2} c\right)$ is the energy density of the external radiation field, which at distances $\leqslant 100 R_{\mathrm{g}}$ is very likely to be dominated by rescattering of disk radiation by electrons in the accretion disk corona of covering factor $\xi_{\mathrm{b}}$. The energy spectrum of the external radiation is approximated by a broken power-law distribution $u_{\text {ext,b }}(E) \propto E^{-\alpha_{\mathrm{i}}}$ with $\alpha_{1}=0, \alpha_{2}=1.8$, and $E_{\mathrm{br}}=10 \mathrm{eV}$ (Richards et al. 2006; Shang et al. 2011). The electron flux is $\dot{N}_{\mathrm{e}, \mathrm{b}} \sim N_{\mathrm{e}, \mathrm{b}} c / r_{\mathrm{b}}$. Assuming that it matches the electron flux in the blazar zone $\left(\dot{N}_{\mathrm{e}, \mathrm{b}} \sim \dot{N}_{\mathrm{e}}\right)$, we calculate the total number of electrons producing the bulk-Compton component to be $N_{\mathrm{e}, \mathrm{b}} \sim 2.4 \times 10^{54}$. We find that the X-ray spectrum of luminosity $L_{\mathrm{b}} \sim 8.7 \times 10^{46} \mathrm{erg} \mathrm{s}^{-1}$ can be reproduced with cold electrons for $\xi_{\mathrm{b}} \simeq 0.19\left(r_{\mathrm{b}} / 6 \times 10^{15} \mathrm{~cm}\right)$.

Multi-wavelength light curves show that X-rays do not correlate with radiation in other spectral bands. This suggests that the $\mathrm{X}$-ray variability of the bulk-Compton radiation can be caused by local wiggling of the jet, e.g., caused by variations of the average direction of non-axisymmetric outflows generated near the $\mathrm{BH}$. Jet wiggling can also explain independent variability in the blazar zone. We have fitted the low state (MJD 54803-5) with an ERCIR model (blue lines in Figure 7) very similar to the one for the high state, changing only the viewing angle, from $\theta_{\text {obs }}=2.3$ to $\theta_{\text {obs }}=3.7$, and the magnetic field strength, from $B^{\prime}=0.22 \mathrm{G}$ to $B^{\prime}=0.20 \mathrm{G}$. With the new viewing angle, the observer is placed outside the jet opening cone and the observed luminosity decreases due to a lower effective Doppler factor. The change in the magnetic field strength reduces the synchrotron luminosity by $\sim 30 \%$ relative to the ERC luminosity. The overall spectral shape is matched without any adjustment in the electron energy distribution.

\section{DISCUSSION}

Multi-wavelength observations of blazars, including $\mathrm{AO}$ $0235+164$, show that events associated with periods of greater activity occur over the entire electromagnetic spectrum, from radio to $\gamma$-rays. Light curves taken in different spectral bands correlate on timescales longer than a month, while on shorter timescales correlations are weaker and some lags are claimed. In particular, monthly lags of the radio signals following the $\gamma$-rays are observed (Pushkarev et al. 2010). This is interpreted in terms of the synchrotron opacity at radio frequencies and indicates that $\gamma$-rays are produced at distances from the $\mathrm{BH}$ that are several parsecs smaller than the position of the radio cores. Whether $\gamma$-ray emission is smoothly distributed over several decades of distance (Blandford \& Levinson 1995), or is associated with specific locations of energy dissipation in a jet, is still debated. The localized dissipation zones could be related to: reconnection of magnetic fields which may operate efficiently at distances of $<0.01 \mathrm{pc}$, where the magnetization parameter $\sigma$ is expected to be large (Nalewajko et al. 2011); internal shocks resulting from collisions between the jet portions moving with different velocities (Spada et al. 2001), which become efficient at distances at which $\sigma$ drops below 0.1 ; oblique/reconfinement shocks which are formed at distances at which interactions of supersonic jets start to feel the influence of the external medium (Hughes et al. 2011; Daly \& Marscher 1988; Komissarov \& Falle 1997; Nalewajko \& Sikora 2009).

Using the SED of AO 0235+164, we showed in Section 6 that the spectrum of this object can be reproduced using a model where the dissipation takes place at a distance of $r \sim 1.7 \mathrm{pc}$ from the $\mathrm{BH}$ and production of $\gamma$-rays is dominated by the Comptonization of NIR radiation of hot dust. The activity of AO $0235+164$ during the same epoch was analyzed independently by Agudo et al. (2011b). They included the VLBI imaging data, and concluded that the $\gamma$-ray emission zone is associated with the $7 \mathrm{~mm}$ radio core (i.e., at $\sim 12$ pc from the $\mathrm{BH}$ ) and propose that production of $\gamma$-rays is dominated by a turbulent multi-zone SSC process. We comment on these differences below, noting 
that we perform detailed modeling of the broadband spectrum of the object.

As the long term radio light curves of AO $0235+164$ indicate, the active season in 2008 started about 200 days before reaching the maximum. During this period of time, any portion of the jet propagates over a distance of $\Delta r \sim c \Gamma^{2} t_{\text {obs }} /(1+z) \geqslant$ tens of parsecs, i.e., much larger than the length of a "blazar zone" inferred from the timescales of the short term flares. Such flares are very likely to be produced by inhomogeneities of the flow, radiating when passing through the region where energy dissipation is intensified. Particularly prominent flares are seen in the optical light curves. Their $\sim 10$ day timescales imply that the extension of the dissipative zone is

$$
\begin{aligned}
\Delta r & \sim \frac{c t_{\mathrm{fl}}}{(1+z)\left(1-\beta \cos \theta_{\mathrm{obs}}\right)} \\
& \sim 1.7 \mathrm{pc}\left(\frac{t_{\mathrm{fl}}}{10 \text { days }}\right)\left(\frac{\Gamma}{20}\right)^{2}(\mathcal{D} / \Gamma),
\end{aligned}
$$

which nicely corresponds with the location of the blazar zone derived from our model. In order to form such flares at a distance of $12 \mathrm{pc}$, a Lorentz factor of $\Gamma \sim 50$ is required. Interestingly, a similarly large Lorentz factor is required in the Agudo et al. (2011b) model to explain the observed $t<20$ day timescale of the flux decay at $\lambda=1 \mathrm{~mm}$. This can be inferred by taking into account that radiative cooling of electrons emitting at $1 \mathrm{~mm}$ is inefficient and that the timescale of the flux decrease, as determined by the adiabatic losses, is $t_{\mathrm{ad}} \simeq(R / c)(1+z) /\left(\theta_{j} \Gamma \mathcal{D}\right)$. VLBI observations of AO $0235+164$ do not exclude such a large value of the bulk Lorentz factor (Jorstad et al. 2001; Piner et al. 2006).

Finally, we comment about the objections made by Agudo et al. (2011b) regarding the application of ERC models for the production of $\gamma$-rays in AO $0235+164$. They pointed out that in this model it is impossible to explain the lack of correlation between short-term variations of the $\gamma$-ray and optical fluxes because of a lack of variations of the external radiation field. However, in the fast cooling regime, variations of the inverse Compton flux are determined not by variations of the seed radiation field, but by variations of the electron injection function. It does not matter whether the seed radiation is external or internal, so this criticism may also be applied to the SSC models. Hence, the lack of a clear correspondence between variations in these two spectral bands must have a different origin than fluctuations in the background radiation. They can be related to variations of the Doppler factor and magnetic fields in the kinematically and geometrically complex dissipative zone. In particular, this can be the case if such a zone is associated with the oblique and/or reconfinement shocks, which in AO $0235+164$ is indicated by the roughly perpendicular orientation of the optical EVPA with respect to the jet axis (see Section 4.3).

\section{CONCLUSIONS}

Fermi-LAT detected enhanced activity in the high-redshift BL Lac object AO 0235+164 during the first six months of operations. We present the results of an intensive multiwavelength campaign covering radio, $\mathrm{mm}$, near-IR, optical, UV, and X-ray bands, as well as optical polarimetry. Extinction in the optical/UV/X-ray band, complicated by the existence of an additional absorbing system at intermediate redshift, has been carefully taken into account. We proposed a modification to the extinction model introduced by Junkkarinen et al. (2004) and used by Raiteri et al. (2005) that corrects a spurious spectral feature in the FUV band.

The $\gamma$-ray spectrum is consistent with a broken power law. Hints of spectral variability can be seen in episodic increases of the $(1-100 \mathrm{GeV}) /(0.1-1 \mathrm{GeV})$ hardness ratio. The brightest $\gamma$-ray flare is much more pronounced in the $0.1-1 \mathrm{GeV}$ energy band.

The $\gamma$-ray activity is roughly correlated with the activity in the optical/near-IR band. There is a possible delay of 15 days of the $R$-band flux with respect to the $\gamma$-ray flux. The optical flux is also correlated with the optical polarization degree, which reaches values up to $35 \%$. At the same time, the optical polarization angle is close to $100^{\circ}$ with moderate scatter. As is typical for blazars, the activity in the radio band is smoother and begins months before the optical $/ \gamma$-ray activity, while the radio-flux peaks are delayed by several weeks with respect to the higher energy bands.

The behavior of the source in the X-ray band is distinct from other bands, as it shows a 20 day high state delayed by a month from the main optical $/ \gamma$-ray flare. The X-ray spectrum during the high state is unusually soft, $\Gamma \sim 2.6$, and is inconsistent with the extrapolation of the optical/UV spectrum, unless we assume a much stronger extinction. We interpret this X-ray component as the bulk-Compton emission, i.e., Comptonization of the accretion-disk radiation reprocessed at the distance of $\sim 100 R_{\mathrm{g}}$, in the region of ongoing jet acceleration and collimation. Such a feature has been tentatively reported before in a few sources, however the present case is still not definitive. The short duration of the high X-ray state can be explained by a rapid "wiggling" of the inner jet.

The broadband SEDs extracted for two different activity states are, with the exception of the X-ray feature, typical for luminous blazars. We interpret the broadband SEDs in the standard leptonic scenario, with the low-energy bump due to synchrotron radiation and the high-energy bump due to Comptonization of the external infrared radiation from the dusty torus (ERCIR). The energetic constraints are very tight, because, if the jet power is comparable to the Eddington luminosity of the central $\mathrm{BH}$, the required radiative efficiency of the jet is $\sim 20 \%$, the magnetization is $\sigma_{\mathrm{B}} \sim 11 \%$, and the pair-to-proton ratio is $n_{\mathrm{e}} / n_{\mathrm{p}} \sim 9$. The bulk Compton feature in the high X-ray state requires, if the electron number flux is to be matched to the model of the flaring state, a covering factor of the accretion disk corona $\xi_{\mathrm{b}} \sim 19 \%$. An alternative interpretation of the highenergy bump with the SSC emission requires a very low covering factor for the dusty torus, in conflict with the observations of quasars.

The Fermi LAT Collaboration acknowledges generous ongoing support from a number of agencies and institutes that have supported both the development and the operation of the LAT as well as scientific data analysis. These include the National Aeronautics and Space Administration and the Department of Energy in the United States, the Commissariat à l'Energie Atomique and the Centre National de la Recherche Scientifique/Institut National de Physique Nucléaire et de Physique des Particules in France, the Agenzia Spaziale Italiana and the Istituto Nazionale di Fisica Nucleare in Italy, the Ministry of Education, Culture, Sports, Science and Technology (MEXT), High Energy Accelerator Research Organization (KEK) and Japan Aerospace Exploration Agency (JAXA) in Japan, and the K. A. Wallenberg Foundation, the Swedish Research Council and the Swedish National Space Board in Sweden. 
Additional support for science analysis during the operations phase is gratefully acknowledged from the Istituto Nazionale di Astrofisica in Italy and the Centre National d'Etudes Spatiales in France.

We acknowledge the support by the Polish MNiSW grant $\mathrm{N}$ N203 301635.

L. C. Reyes acknowledges support from NASA through Swift Guest Investigator Grant NNX10AJ70G; as well as support by the Kavli Institute for Cosmological Physics at the University of Chicago through grants NSF PHY-0114422 and NSF PHY-0551142 and an endowment from the Kavli Foundation and its founder Fred Kavli.

This research is partly based on observations with the $100 \mathrm{~m}$ telescope of the MPIfR (Max-Planck-Institut für Radioastronomie) at Effelsberg. This work has made use of observations with the IRAM 30 m telescope.

This paper is partly based on observations carried out at the German-Spanish Calar Alto Observatory, which is jointly operated by the MPIA and the IAA-CSIC.

The Abastumani team acknowledges financial support by the Georgian National Science Foundation through grant GNSF/ST08/4-404.

The Metsähovi team acknowledges the support from the Academy of Finland to our observing projects (numbers 212656 , 210338, 121148, and others).

The Submillimeter Array is a joint project between the Smithsonian Astrophysical Observatory and the Academia Sinica Institute of Astronomy and Astrophysics and is funded by the Smithsonian Institution and the Academia Sinica.

The acquisition and analysis of the SMARTS data are supported by Fermi GI grants 011283 and 31155 (PI: C. Bailyn).

Data from the Steward Observatory spectropolarimetric monitoring project were used. This program is supported by Fermi Guest Investigator grants NNX08AW56G and NNX09AU10G.

UMRAO research is supported by a series of grants from the NSF and NASA, most recently AST-0607523 and Fermi GI grants NNX10AP16G and NNX11AO13G, respectively; funds for telescope operation are provided by the University of Michigan.

\section{REFERENCES}

Abdo, A. A., Ackermann, M., Ajello, M., et al. 2009, ApJS, 183, 46 Abdo, A. A., Ackermann, M., Ajello, M., et al. 2010a, ApJS, 188, 405 Abdo, A. A., Ackermann, M., Ajello, M., et al. 2010b, ApJ, 710, 1271 Agudo, I., Jorstad, S. G., Marscher, A. P., et al. 2011a, ApJ, 726, L13 Agudo, I., Marscher, A. P., Jorstad, S. G., et al. 2011b, ApJ, 735, L10 Angelakis, E., Fuhrmann, L., Marchili, N., Krichbaum, T. P., \& Zensus, J. A. 2008, Mem. Soc. Astronom. Ital., 75, 282

Atwood, W. B., Abdo, A. A., Ackermann, M., et al. 2009, ApJ, 697, 1071 Baars, J. W. M., Genzel, R., Pauliny-Toth, I. I. K., \& Witzel, A. 1977, A\&A, 61,99

Bach, U., Gurwell, M. A., Leto, P., et al. 2008, ATel, 1849

Baldwin, J. A., \& Stone, R. P. S. 1984, MNRAS, 206, 241

Begelman, M. C., \& Sikora, M. 1987, ApJ, 322, 650

Blandford, R. D., \& Leninson, A. 1995, ApJ, 441, 79

Blandford, R. D., \& Rees, M. J. 1978, in Pittsburgh Conference on BL Lac Objects, ed. A. M. Wolfe (Pittsburgh: Univ. Pittsburgh Press), 328 Böttcher, M. 2007, Ap\&SS, 309, 95

Breeveld, A. A. 2011, in Proc. of AIP Conf. Ser. Vol. 1358, An Updated Ultraviolet Calibration for the Swift/UVOT (New York: AIP) 1358, 373

Burbidge, E. M., Caldwell, R. D., Smith, H. E., Liebert, J., \& Spinrad, H. 1976, ApJ, 205, L117

Burrows, D. N., Hill, J. E., Nousek, J. A., et al. 2005, Space Sci. Rev., 120, 165

Cardelli, J. A., Clayton, G. C., \& Mathis, J. S. 1989, ApJ, 345, 245

Celotti, A., Ghisellini, G., \& Fabian, A. C. 2007, MNRAS, 375, 417

Chu, H. S., Baath, L. B., Rantakyro, F. T., et al. 1996, A\&A, 307, 15

Cohen, R. D., Smith, H. E., Junkkarinen, V. T., et al. 1987, ApJ, 318, 577
Comastri, A., Fossati, G., Ghisellini, G., \& Molendi, S. 1997, ApJ, 480, 534

Corbel, S., \& Reyes, L. C. 2008, ATel, 1744

Corbett, E. A., Robinson, A., Axon, D. J., \& Hough, J. H. 2000, MNRAS, 311 , 485

Cutri, R. M., Skrutskie, M. F., van Dyk, S., et al. 2003, VizieR On-line Data Catalog, http://adsabs.harvard.edu/abs/2003yCat.2246....0C

Daly, R. A., \& Marscher, A. P. 1988, ApJ, 334, 539

DePoy, D. L., Atwood, B., Belville, S. R., et al. 2003, Proc. SPIE, 4841, 827

D’Ammando, F., Pucella, G., Raiteri, C. M., et al. 2009, A\&A, 508, 181

Edelson, R. A., \& Krolik, J. H. 1988, ApJ, 333, 646

Elvis, M., Wilkes, B. J., McDowell, J. C., et al. 1994, ApJS, 95, 1

Foschini, L., Iafrate, G., \& Longo, F. 2008, ATel, 1784

Francis, P. J., Hewett, P. C., Foltz, C. B., et al. 1991, ApJ, 373, 465

Fuhrmann, L., Krichbaum, T. P., Witzel, A., et al. 2008, A\&A, 490, 1019

Fuhrmann, L., Zensus, J. A., Krichbaum, T. P., Angelakis, E., \& Readhead, A. C. S. 2007, in AIP Conf. Proc. 921, The First GLAST Symposium (Melville, NY: AIP), 249

Gehrels, N., Chincarini, G., Giommi, P., et al. 2004, ApJ, 611, 1005

Ghosh, K. K., \& Soundararajaperumal, S. 1995, ApJS, 100, 37

Gonzalez-Perez, J. N., Kidger, M. R., \& Martin-Luis, F. 2001, AJ, 122, 2055

Haas, M., Müller, S. A. H., Bertoldi, F., et al. 2004, A\&A, 424, 531

Hagen-Thorn, V. A., Larionov, V. M., Jorstad, S. G., et al. 2008, ApJ, 672, 40

Hughes, P. A., Aller, M. F., \& Aller, H. D. 2011, ApJ, 735, 81

Hunter, S. D., Bertsch, D. L., Dingus, B. L., et al. 1993, ApJ, 409, 134

Jones, D. L., Unwin, S. C., Baath, L. B., \& Davis, M. M. 1984, ApJ, 284, 60

Jorstad, S. G., Marscher, A. P., Mattox, J. R., et al. 2001, ApJS, 134, 181

Junkkarinen, V. T., Cohen, R. D., Beaver, E. A., et al. 2004, ApJ, 614, 658

Komissarov, S. S., Barkov, M. V., Vlahakis, N., \& Königl, A. 2007, MNRAS, 380,51

Komissarov, S. S., \& Falle, S. A. E. G. 1997, MNRAS, 288, 833

Landolt, A. U. 1992, ApJ, 104, 340

Levinson, A. 2006, Int. J. Mod. Phys. A, 21, 6015

Liu, F. K., Zhao, G., \& Wu, X.-B. 2006, ApJ, 650, 749

Madejski, G., Takahashi, T., Tashiro, M., et al. 1996, ApJ, 459, 156

Massey, P., Strobel, K., Barnes, J. V., \& Anderson, E. 1988, ApJ, 328, 315

Mattox, J. L., et al. 1996, ApJ, 461, 396

Moderski, R., Sikora, M., \& Błażejowski, M. 2003, A\&A, 406, 855

Murphy, D. W., Browne, I. W. A., \& Perley, R. A. 1993, MNRAS, 264, 298

Nalewajko, K. 2009, MNRAS, 395, 524

Nalewajko, K., Giannios, D., Begelman, M. C., et al. 2011, MNRAS, 413, 333

Nalewajko, K., \& Sikora, M. 2009, MNRAS, 392, 1205

Nilsson, K., Charles, P. A., Pursimo, T., et al. 1996, A\&A, 314, 754

Padovani, P., Costamante, L., Giommi, P., et al. 2004, MNRAS, 347, 1282

Pei, Y. C. 1992, ApJ, 395, 130

Persson, S. E., Murphy, D. C., Krzeminski, W., Roth, M., \& Rieke, M. J. 1998, AJ, 116, 2475

Piner, B. D., Bhattarai, D., Edwards, P. G., \& Jones, D. L. 2006, ApJ, 640, 196 Poole, T. S., Breeveld, A. A., Page, M. J., et al. 2008, MNRAS, 383, 627 Pushkarev, A. B., Kovalev, Y. Y., \& Lister, M. L. 2010, ApJ, 722, L7 Raiteri, C. M., Villata, M., Aller, H. D., et al. 2001, A\&A, 377, 396

Raiteri, C. M., Villata, M., Bruschini, L., et al. 2010, A\&A, 524, A43 Raiteri, C. M., Villata, M., Capetti, A., et al. 2007, A\&A, 464, 871

Raiteri, C. M., Villata, M., Ibrahimov, M. A., et al. 2005, A\&A, 438, 39

Raiteri, C. M., Villata, M., Kadler, M., et al. 2006, A\&A, 459, 731

Raiteri, C. M., Villata, M., Larionov, V. M., et al. 2008, A\&A, 480, 339

Ravasio, M., Tagliaferri, G., Ghisellini, G., et al. 2003, A\&A, 408, 479

Richards, G. T., Lacy, M., Storrie-Lombardi, L. J., et al. 2006, ApJS, 166, 470

Richards, J., Max-Moerbeck, W., Pavlidou, V., et al. 2011, ApJS, 194, 29

Rieke, G. H., Grasdalen, G. L., Kinman, T. D., et al. 1976, Nature, 260, 754

Roberts, M. S., Brown, R. L., Brundage, W. D., et al. 1976, AJ, 81, 293

Roming, P. W. A., Kennedy, T. E., Mason, K. O., et al. 2005, Space Sci. Rev., 120,95

Sasada, M., Uemura, M., Fukazawa, Y., et al. 2011, PASJ, 63, 489

Schlegel, D. J., Finkbeiner, D. P., \& Davis, M. 1998, ApJS, 500, 525

Schmidt, G. D., Elston, R., \& Lupie, O. L. 1992a, AJ, 104, 1563

Schmidt, G. D., Stockman, H. S., \& Smith, P. S. 1992b, ApJ, 398, L57

Shang, Z., Brotherton, M. S., Wills, B. J., et al. 2011, ApJS, 196, 2

Sikora, M. 2011, in IAU Symp. 275, Jets at All Scales, ed. G. E. Romero, R. A. Sunyaev, \& T. Belloni (Cambridge: Cambridge Univ. Press), 59

Sikora, M., Begelman, M. C., Madejski, G. M., \& Lasota, J.-P. 2005, ApJ, 625, 72

Sikora, M., \& Madejski, G. 2000, ApJ, 534, 109

Sikora, M., \& Madejski, G. 2001, Am. Inst. Phys. Conf. Ser., 558, 275

Sikora, M., Stawarz, Ł., Moderski, R., et al. 2009, ApJ, 704, 38

Snijders, M. A. J., Bocksenberg, A., Penston, M. V., \& Sargent, W. L. W. 1982, MNRAS, 201, 801 
Smith, P. S., Montiel, E., Rightley, S., et al. 2009, Fermi Symposium, eConf Proceedings C091122 (arXiv:0912.3621)

Smith, P. S., Schmidt, G. D., Hines, D. C., \& Foltz, C. B. 2003, ApJ, 593, 676

Spada, M., Ghisellini, G., Lazzati, D., \& Celotti, A. 2001, MNRAS, 325, 1559

Spearman, C. 1904, Am. J. Psychol., 15, 72101

Spinrad, H., \& Smith, H. 1975, ApJ, 201, 275

Stein, W. A., O’Dell, S. L., \& Strittmatter, P. A. 1976, ARA\&A, 14, 173

Stickel, M., Padovani, P., Urry, C. M., Fried, J. W., \& Kuehr, H. 1991, ApJ, 374, 431

Stocke, J. T., Danforth, C. W., \& Perlman, E. S. 2011, ApJ, 732, 1312

Stone, R. P. S., \& Baldwin, J. A. 1983, MNRAS, 204, 347

Trippe, S., Neri, R., Krips, M., et al. 2010, A\&A, 515, A40

Tristram, K. R. W., \& Schartmann, M. 2011, A\&A, 531, A99

Urry, C. M. 1999, in ASP Conf. Ser. 159, BL Lac Phenomenon, ed. L. O. Takalo \& A. Sillanpää (San Francisco, CA: ASP), 3
Vermeulen, R. C., \& Taylor, G. B. 1995, AJ, 109, 1983

Villata, M., Raiteri, C. M., Carosati, D., et al. 2008a, ATel, 1724

Villata, M., Raiteri, C. M., Gurwell, M. A., et al. 2009, A\&A, 504, L9

Villata, M., Raiteri, C. M., Larionov, V. M., et al. 2008b, A\&A, 481, L79

Villata, M., Raiteri, C. M., Larionov, V. M., et al. 2008c, ATel, 1785

Wardle, J. F. C., \& Kronberg, P. P. 1974, ApJ, 194, 249

Watanabe, M., Nakaya, H., Yamamuro, T., et al. 2005, PASP, 117, 870

Webb, J. R., Howard, E., Benítez, E., et al. 2000, AJ, 120, 41

Winters, J. M., \& Neri, R. 2010, An Introduction to the IRAM Plateau de Bure Interferometer, Public IRAM Document, Version 4.1-01

Wolfe, A. M., \& Wills, B. 1977, ApJ, 218, 39

Wolff, M. J., Nordsieck, K. H., \& Nook, M. A. 1996, AJ, 111, 856

Worral, D. M., \& Wilkes, B. J. 1990, ApJ, 360, 396

Wu, X.-B., Liu, F. K., Kong, M. Z., et al. 2011, J. Astrophys. Astron., 32, 209 